\title{
$p$-ADIC EISENSTEIN-KRONECKER SERIES FOR CM ELLIPTIC CURVES AND THE KRONECKER LIMIT FORMULAS
}

\author{
KENICHI BANNAI, HIDEKAZU FURUSHO, \\ AND SHINICHI KOBAYASHI
}

\begin{abstract}
Consider an elliptic curve defined over an imaginary quadratic field $K$ with good reduction at the primes above $p \geq 5$ and with complex multiplication by the full ring of integers $\mathcal{O}_{K}$ of $K$. In this paper, we construct $p$-adic analogues of the Eisenstein-Kronecker series for such an elliptic curve as Coleman functions on the elliptic curve. We then prove $p$-adic analogues of the first and second Kronecker limit formulas by using the distribution relation of the Kronecker theta function.
\end{abstract}

\section{$\S 1$. Introduction}

Let $\Gamma \subset \mathbb{C}$ be a lattice. Then for an integer $a$ and $z, w \in \mathbb{C}$, the EisensteinKronecker-Lerch series for the lattice $\Gamma$ is defined by

$$
K_{a}^{*}(z, w, s ; \Gamma)=\sum_{\gamma \in \Gamma \backslash\{-z\}} \frac{(\bar{z}+\bar{\gamma})^{a}}{|z+\gamma|^{2 s}} \chi_{w}(\gamma),
$$

where $A(\Gamma)$ is the area of the fundamental domain of $\Gamma$ divided by $\boldsymbol{\pi}=$ $3.1415 \ldots$ and $\chi_{w}(z):=\exp [(z \bar{w}-w \bar{z}) / A(\Gamma)]$ for any $z, w \in \mathbb{C}$. The above series converges for $\operatorname{Re}(s)>a / 2+1$, but one may give it meaning for general $s$ by analytic continuation. In what follows, we will omit $\Gamma$ from the notation if there is no fear of confusion. For integers $m, n$, the classical EisensteinKronecker function, more commonly known as the Kronecker double series, is defined to be the function

$$
E_{m, n}(z):=K_{n-m}^{*}(0, z, n) .
$$

Received June 28, 2012. Revised June 18, 2014. Accepted August 14, 2014.

2010 Mathematics Subject Classification. Primary 11G55; Secondary 11G07, 11G15, 14F30, 14G10. 
In the context of polylogarithms, these functions are elliptic analogues of the Bloch-Wigner-Zagier polylogarithm function (see [21, Remark, p. 282]). In the paper [14], Coleman defined the $p$-adic analogue of the classical polylogarithm function as a Coleman function, which is a class of $p$-adic analytic functions generalizing the rigid analytic functions. The purpose of this paper is to define for integers $m, n$ the $p$-adic analogue $E_{m, n}^{c o l}(z)$ of the EisensteinKronecker function as a Coleman function, when the complex torus $\mathbb{C} / \Gamma$ has a model as an elliptic curve defined over an imaginary quadratic field $K$ with good reduction at the primes above $p \geq 5$ and complex multiplication by the full ring of integers $\mathcal{O}_{K}$ of $K$. The main ingredient in the construction of the Eisenstein-Kronecker series is the distribution relation.

Focusing on the distribution relation of the Eisenstein-Kronecker series, we then prove the $p$-adic analogues of the Kronecker limit formulas. We let $\theta(z)$ be the reduced theta function on $\mathbb{C} / \Gamma$ associated to the divisor $[0] \subset$ $\mathbb{C} / \Gamma$, normalized so that $\theta^{\prime}(0)=1$. (See (2) for the precise transformation formula.) Then the Kronecker limit formulas in the classical complex case are given as follows.

THEOREM 1.1 (Kronecker limit formulas). Let $c$ be the Euler constant $c:=\lim _{n \rightarrow \infty}(1+1 / 2+\cdots+1 / n-\log n)$, and let $\Delta$ be the discriminant of $\Gamma$ defined as $\Delta:=g_{2}^{3}-27 g_{3}^{2}$, where $g_{k}:=\sum_{\gamma \in \Gamma \backslash\{0\}} \gamma^{-2 k}$. Then we have the following.

(i) The first limit formula

$$
\lim _{s \rightarrow 1}\left(A K_{0}^{*}(0,0, s)-\frac{1}{s-1}\right)=-\frac{1}{12} \log |\Delta|^{2}-2 \log A+2 c .
$$

(ii) For $z \notin \Gamma$, the second limit formula

$$
A K_{0}^{*}(0, z, 1)=-\log |\theta(z)|^{2}+\frac{|z|^{2}}{A}-\frac{1}{12} \log |\Delta|^{2} .
$$

Numerous proofs exist for the classical case, but many of the proofs rely on arguments concerning the moduli space. We give a new proof of the above theorem, valid for a fixed lattice $\Gamma \subset \mathbb{C}$, using the Kronecker theta function and the distribution relation. Our view of understanding the Kronecker limit formulas in terms of the Kronecker theta function and the distribution relation allows us to prove the $p$-adic analogues of Theorem 1.1. Suppose now that $\Gamma$ corresponds to a period lattice corresponding to the invariant differential $\omega=d x / y$ of an elliptic curve $E: y^{2}=4 x^{3}-g_{2} x-g_{3}$ with complex 
multiplication by the ring of integers $\mathcal{O}_{K}$ of an imaginary quadratic field $K$. We assume in addition that $E$ is defined over $K$, and that the model above has good reduction at the primes above $p \geq 5$. We denote

$$
K_{n-m}^{\mathrm{col}}(0, z, n):=E_{m, n}^{\mathrm{col}}(z)
$$

to highlight the analogy. Then in analogy with Theorem 1.1(ii), we have the following.

Theorem 1.2 ( $p$-adic second Kronecker limit formula). For any prime $p \geq 5$ of good reduction, we have the second limit formula

$$
K_{0}^{\mathrm{col}}(0, z, 1)=-\log _{p} \theta(z)-\frac{1}{12} \log _{p} \Delta
$$

where $\log _{p} \theta(z)$ is a certain $p$-adic analogue of the function $\log |\theta(z)|-|z|^{2} / A$ defined in Definition 5.1 using the reduced theta function $\theta(z)$ and the branch of our p-adic logarithm.

The $p$-adic analogues of the second Kronecker limit formula were previously investigated by Katz [20] and de Shalit [18] in the context of $p$-adic $L$-functions when $p$ is a prime of good ordinary reduction. Our formulation via the $p$-adic Eisenstein-Kronecker series gives a direct $p$-adic analogue, and is valid even for supersingular $p$.

Suppose now that $p \geq 5$ is a prime of good ordinary reduction. In this case, the prime $p$ splits as $p=\mathfrak{p p}^{*}$ in $\mathcal{O}_{K}$. We denote by $\psi$ the Hecke character of $K$ associated to $E$, and we let $\pi:=\psi(\mathfrak{p})$. In [5, Section 3.1], we defined a two-variable $p$-adic measure $\mu:=\mu_{0,0}$ on $\mathbb{Z}_{p} \times \mathbb{Z}_{p}$ interpolating EisensteinKronecker numbers, or more precisely, the values $K_{a+b}^{*}(0,0, b) / A^{a}$ for $a$, $b \geq 0$. This measure depends on the choice of a $p$-adic period $\Omega_{p}$ of the formal group of $E$. We define the $p$-adic Eisenstein-Kronecker-Lerch series by

$$
K_{a}^{(p)}(0,0, s):=\int_{\mathbb{Z}_{p}^{\times} \times \mathbb{Z}_{p}^{\times}}\langle x\rangle^{s-1} \omega(y)^{a-1}\langle y\rangle^{a-s} d \mu(x, y)
$$

for any $s \in \mathbb{Z}_{p}$, where $\langle-\rangle: \mathbb{Z}_{p}^{\times} \rightarrow \mathbb{C}_{p}^{\times}$is given as the composition $\mathbb{Z}_{p}^{\times} \rightarrow$ $1+p \mathbb{Z}_{p} \hookrightarrow \mathbb{C}_{p}^{\times}$and $\omega: \mathbb{Z}_{p}^{\times} \rightarrow \mu_{p-1}$ is the Teichmüller character, so that $x=\omega(x)\langle x\rangle$ for any $x \in \mathbb{Z}_{p}^{\times}$. Then an argument similar to the proof of Theorem 1.1(i) gives the following. 
Proposition 1.3 ( $p$-adic first Kronecker limit formula). Suppose that $p \geq 5$ is a prime of good ordinary reduction. Then

$$
\lim _{s \rightarrow 1} K_{0}^{(p)}(0,0, s)=\Omega_{p}^{-1}\left(1-\frac{1}{p}\right) \log _{p} \bar{\pi},
$$

where $\Omega_{p}$ is the $p$-adic period corresponding to $\mu$.

The proof of the above proposition is parallel to that of the proof of Theorem 1.1(i). However, due to the existence of a trivial zero for the function $K_{0}^{(p)}(0,0, s)$ at $s=1$, the analogy with the classical case is not perfect. (See Remark 5.8 for details.)

The $p$-adic analogue of the classical polylogarithm was used to express the specializations at $p$-power roots of unity of the $p$-adic realization of the polylogarithm sheaf (see [17], [23], [10], [11], [13], [1], [3]). The elliptic analogue of the classical polylogarithm sheaf was first constructed by Beilinson and Levin [8]. In previous research, we studied the $p$-adic realization of the elliptic polylogarithm sheaf for CM elliptic curves (see [2], $[7],[6])$. As in the classical case, the $p$-adic Eisenstein-Kronecker function defined in this paper should be related to specializations at $p$-power torsion points of the $p$-adic realization of the elliptic polylogarithm sheaf. We expect that this function will play a role in future research in the formulation of the $p$-adic analogue of the elliptic Zagier conjecture formulated by Wildeshaus [25].

\section{§2. Classical Kronecker limit formulas}

In this section, we first review the definitions of the Eisenstein-Kronecker series and the Kronecker theta function. We then give new proofs of the first and second Kronecker limit formulas using the Kronecker theta function and the distribution relation. Our proof in the classical complex case will be the model for proving the $p$-adic analogue. As in the original proof by Kronecker, we first prove the second limit formula, and then deduce the first limit formula from the second.

\subsection{Eisenstein-Kronecker series and the Kronecker theta func- tion}

We fix a lattice $\Gamma$ in $\mathbb{C}$, and we let $A$ be the area of the fundamental domain of $\Gamma$ divided by $\pi$. Let $a$ be an integer, and let $z_{0}, w_{0} \in \mathbb{C}$. We denote by $K_{a}^{*}\left(z_{0}, w_{0}, s\right)$ the Eisenstein-Kronecker-Lerch series given in (1) 
of the Introduction. The following result was proved by Weil (see also [7, Proposition 2.4] for details concerning the case $a \leq 0$ ).

Proposition 2.1 ([24, Chapter VIII, Section 13]). Let a be an integer.

(i) The function $K_{a}^{*}\left(z_{0}, w_{0}, s\right)$ for $s$ continues meromorphically to a function on the whole $s$ plane, with a simple pole only at $s=1$ if $a=0$ and $w_{0} \in \Gamma$.

(ii) The function $K_{a}^{*}\left(z_{0}, w_{0}, s\right)$ satisfies the functional equation

$$
\Gamma(s) K_{a}^{*}\left(z_{0}, w_{0}, s\right)=A^{a+1-2 s} \Gamma(a+1-s) K_{a}^{*}\left(w_{0}, z_{0}, a+1-s\right) \chi_{w_{0}}\left(z_{0}\right) .
$$

As in the Introduction, we define the Eisenstein-Kronecker function, referred to more commonly as the Kronecker double series, as follows.

Definition 2.2. For any integer $m, n$, we define the Eisenstein-Kronecker function $E_{m, n}(z)$ to be the $\mathscr{C}^{\infty}$-function on $\mathbb{C} \backslash \Gamma$ defined by

$$
E_{m, n}(z):=K_{n-m}^{*}(0, z, n)
$$

This function is known to satisfy the differential equations

$$
\partial_{z} E_{m, n}(z)=-E_{m-1, n}(z) / A, \quad \partial_{\bar{z}} E_{m, n}(z)=E_{m, n-1}(z) / A .
$$

REMARK 2.3. The Eisenstein-Kronecker functions $E_{m, n}(z)$ may be used to describe the $\mathbb{R}$-Hodge realization of the elliptic polylogarithm sheaf (see [7, Appendix] for details).

We next review the definition and basic properties of the Kronecker theta function. Denote by $\theta(z)$ the reduced theta function associated to the divisor $[0]$ of $\mathbb{C} / \Gamma$, normalized so that $\theta^{\prime}(0)=1$. This is the function used by Robert to define his elliptic units (see [22, Section 1]). This function satisfies the transformation formula

$$
\theta(z+\gamma)=\alpha(\gamma) \exp \left(\frac{z \gamma}{A}+\frac{|\gamma|^{2}}{2 A}\right) \theta(z)
$$

where $\alpha(\gamma)=-1$ if $\gamma \notin 2 \Gamma$ and $\alpha(\gamma)=1$ otherwise. We define the Kronecker theta function as follows.

Definition 2.4 (Kronecker theta function). We let

$$
\Theta(z, w):=\frac{\theta(z+w)}{\theta(z) \theta(w)} .
$$


The relation of this function to the two-variable Jacobi theta function $F_{\tau}(z, w)$ of Zagier [26] is given by $\Theta(z, w)=\exp (z w / A) F_{\tau}(z, w)$.

The values $K_{a}^{*}(z, w, s)$ are defined for any $z, w \in \mathbb{C}$, but as a function, $K_{a}^{*}(z, w, s)$ is not continuous for $z, w \in \Gamma$. We let $K_{a}(z, w, s):=K_{a}^{*}(z, w, s)$ be the $\mathscr{C}^{\infty}$-function defined for any $z, w \in \mathbb{C} \backslash \Gamma$. We regard the function $K_{a}(z, w, s)$ as being undefined for $z$ or $w \in \Gamma$. Since the function $K_{a}^{*}(z, w, s)$ for $z \in \Gamma$ is defined in (1) by removing the summand with poles, we have

$$
\lim _{z \rightarrow 0}\left[K_{1}(z, w, 1)-\frac{1}{z}\right]=K_{1}^{*}(0, w, 1) .
$$

The relation between this function and the Kronecker theta function is given by the following theorem due to Kronecker.

Theorem 2.5 ([24, Chapter VIII, Section 4(7)]). We have

$$
\Theta(z, w)=\exp \left[\frac{z \bar{w}}{A}\right] K_{1}(z, w, 1) .
$$

The above theorem was originally proved in terms of Jacobi theta functions by Kronecker using moduli arguments (see, e.g., [24]). In [5, Theorem 1.13] or [4, Theorem 2.10], we give another proof valid for a fixed lattice $\Gamma \subset \mathbb{C}$ using the fact that both sides of the equality are reduced meromorphic theta functions associated to the Poincaré bundle on $\mathbb{C} / \Gamma \times \mathbb{C} / \Gamma$, with the same poles and the same residue at each pole.

\subsection{Proof of the second limit formula}

We now deduce Theorem 1.1(ii) from Theorem 2.5.

Proposition 2.6. There exists a constant $C$ such that

$$
\log |\theta(z)|^{2}-\frac{|z|^{2}}{A}=-A K_{0}^{*}(0, z, 1)+C
$$

for any $z \notin \Gamma$.

Proof. By Theorem 2.5, we have

$$
\Theta(z, w)-\frac{1}{z}=\exp \left[\frac{z \bar{w}}{A}\right]\left(K_{1}(z, w, 1)-\frac{1}{z}\right)+\frac{1}{z}\left(\exp \left[\frac{z \bar{w}}{A}\right]-1\right) .
$$

Hence by (3), we have

$$
\lim _{z \rightarrow 0}\left(\Theta(z, w)-\frac{1}{z}\right)=K_{1}^{*}(0, w, 1)+\frac{\bar{w}}{A} .
$$


Direct computation also shows that

$$
\lim _{z \rightarrow 0}\left(\Theta(z, w)-\frac{1}{z}\right)=\frac{\theta^{\prime}(w)}{\theta(w)}
$$

Hence we have

$$
K_{1}^{*}(0, w, 1)+\frac{\bar{w}}{A}=\frac{\theta^{\prime}(w)}{\theta(w)}=\frac{\partial}{\partial w} \log \theta(w)
$$

In particular, if we replace the variable $w$ in the above by $z$, then we have

$$
\frac{\partial}{\partial z}\left(\log \theta(z)-\frac{z \bar{z}}{A}\right)=K_{1}^{*}(0, z, 1)
$$

Therefore, if we let $\Xi(z)$ be the function

$$
\Xi(z):=\log |\theta(z)|^{2}-\frac{|z|^{2}}{A}
$$

then we have

$$
\frac{\partial}{\partial z} \Xi(z)=K_{1}^{*}(0, z, 1), \quad \frac{\partial}{\partial \bar{z}} \Xi(z)=\overline{K_{1}^{*}(0, z, 1)}
$$

On the other hand, one can directly show that

$$
A \frac{\partial}{\partial z} K_{0}^{*}(0, z, 1)=-K_{1}^{*}(0, z, 1), \quad A \frac{\partial}{\partial \bar{z}} K_{0}^{*}(0, z, 1)=-\overline{K_{1}^{*}(0, z, 1)}
$$

(see, e.g., [7, Lemma A.1]). Hence $\Xi(z)+A K_{0}^{*}(0, z, 1)$ must be constant.

Our goal is to determine the constant $C$. We use the following result, which is a type of distribution relation. In what follows, we will write $z_{n} \neq 0$ for $n \geq 1$ to mean $z_{n} \in\left(\frac{1}{n} \Gamma / \Gamma\right) \backslash\{0\}$ for simplicity.

LEMMA 2.7 (Distribution relation). We have

$$
\sum_{z_{n} \neq 0} K_{0}^{*}\left(0, z_{n}, 1\right)=-\frac{2 \log n}{A}
$$

where the sum is over all $n$-torsion points $z_{n}$ of $\mathbb{C} / \Gamma$ except zero. 
Proof. We have

$$
\sum_{z_{n} \in \frac{1}{n} \Gamma / \Gamma} \chi_{z_{n}}(\gamma)= \begin{cases}n^{2} & (\gamma \in n \Gamma) \\ 0 & (\gamma \notin n \Gamma)\end{cases}
$$

Therefore

$$
\frac{1}{n^{2}} \sum_{z_{n} \in \frac{1}{n} \Gamma / \Gamma} K_{0}^{*}\left(0, z_{n}, s\right)=\sum_{\gamma \in n \Gamma \backslash\{0\}} \frac{1}{|\gamma|^{2 s}}=\frac{1}{n^{2 s}} K_{0}^{*}(0,0, s)
$$

when the real part of $s$ is sufficiently large, and hence for any $s$ by analytic continuation. In particular, we have

$$
\frac{1}{n^{2}} \sum_{z_{n} \neq 0} K_{0}^{*}\left(0, z_{n}, s\right)=\left(\frac{1}{n^{2 s}}-\frac{1}{n^{2}}\right) K_{0}^{*}(0,0, s) .
$$

By equation (31) in [24, Chapter VIII, Section 13], noting that $\Gamma(1)=1$, the residue of $K_{0}^{*}(0,0, s)$ at $s=1$ is $1 / A$. Hence we have

$$
\frac{1}{n^{2}} \sum_{z_{n} \neq 0} K_{0}^{*}\left(0, z_{n}, 1\right)=-\frac{2 \log n}{n^{2} A}
$$

as desired.

The above lemma shows that the constant $C$ is

$$
C=\frac{1}{n^{2}-1}\left[\sum_{z_{n} \neq 0}\left(\log \left|\theta\left(z_{n}\right)\right|^{2}-\frac{\left|z_{n}\right|^{2}}{A}\right)-2 \log n\right] .
$$

We will now calculate this value explicitly in terms of $\Delta$.

Proposition 2.8. We have

$$
\frac{1}{4} \log \left|\Delta^{\prime}\right|^{2}=-\sum_{z_{2} \neq 0}\left(\log \left|\theta\left(z_{2}\right)\right|^{2}-\frac{\left|z_{2}\right|^{2}}{A}\right),
$$

where $z_{2}$ runs through nontrivial 2-torsion points of $\mathbb{C} / \Gamma$, and

$$
\Delta^{\prime}=\left(e_{1}-e_{2}\right)^{2}\left(e_{2}-e_{3}\right)^{2}\left(e_{3}-e_{1}\right)^{2}
$$

for $y^{2}=4 x^{3}-g_{2} x-g_{3}=4\left(x-e_{1}\right)\left(x-e_{2}\right)\left(x-e_{3}\right)$. 
Proof. Note that

$$
\left(x-e_{1}\right)\left(x-e_{2}\right)\left(x-e_{3}\right)=\prod_{z_{2} \neq 0}\left(x-\wp\left(z_{2}\right)\right) .
$$

Then if $\Gamma=\mathbb{Z} \omega_{1}+\mathbb{Z} \omega_{2}$, we may suppose that $e_{1}=\wp\left(\omega_{1} / 2\right), e_{2}=\wp\left(\omega_{2} / 2\right)$, and $e_{3}=\wp\left(\left(\omega_{1}+\omega_{2}\right) / 2\right)$. Since

$$
\theta(z+w) \theta(z-w) \theta(z)^{-2} \theta(w)^{-2}=\wp(w)-\wp(z)
$$

we have

$$
\begin{aligned}
& \theta\left(\frac{\omega_{1}+\omega_{2}}{2}\right) \theta\left(\frac{\omega_{1}-\omega_{2}}{2}\right) \theta\left(\frac{\omega_{1}}{2}\right)^{-2} \theta\left(\frac{\omega_{2}}{2}\right)^{-2}=e_{2}-e_{1}, \\
& \theta\left(\omega_{1}+\frac{\omega_{2}}{2}\right) \theta\left(\frac{\omega_{2}}{2}\right) \theta\left(\frac{\omega_{1}+\omega_{2}}{2}\right)^{-2} \theta\left(\frac{\omega_{1}}{2}\right)^{-2}=e_{1}-e_{3}, \\
& \theta\left(\omega_{2}+\frac{\omega_{1}}{2}\right) \theta\left(\frac{\omega_{1}}{2}\right) \theta\left(\frac{\omega_{1}+\omega_{2}}{2}\right)^{-2} \theta\left(\frac{\omega_{2}}{2}\right)^{-2}=e_{2}-e_{3} .
\end{aligned}
$$

Hence using the transformation formula

$$
\theta(z+\gamma)=\alpha(\gamma) \exp \left(\frac{z \bar{\gamma}}{A}+\frac{\gamma \bar{\gamma}}{2 A}\right) \theta(z)
$$

of $\theta(z)$, where $\gamma$ is any element in $\Gamma$ and $\alpha(\gamma)=1$ if $\gamma \in 2 \Gamma$ and $=-1$ otherwise, the value $\Delta^{\prime}$ is

$$
\exp \left[\frac{\omega_{1} \overline{\omega_{1}}+\omega_{2} \overline{\omega_{2}}+\overline{\omega_{1}} \omega_{2}}{A}\right] \theta\left(\frac{\omega_{1}}{2}\right)^{-4} \theta\left(\frac{\omega_{2}}{2}\right)^{-4} \theta\left(\frac{\omega_{1}+\omega_{2}}{2}\right)^{-4}
$$

Multiplying it and its complex conjugation and taking the logarithm, we obtain the formula. Note that since we take the logarithm of positive real numbers, the values do not depend on the choice of the branch of the logarithm.

Proof of Theorem 1.1(2). Since the Ramanujan $\Delta$ is given by $\Delta=2^{4} \Delta^{\prime}$, we have by Lemma 2.7 and Proposition 2.8 that

$$
C=\frac{1}{3}\left(-\frac{1}{4} \log \left|\Delta^{\prime}\right|^{2}-2 \log 2\right)=-\frac{1}{12} \log |\Delta|^{2} .
$$

Our assertion now follows from Proposition 2.6. 


\subsection{Proof of the first limit formula}

We first review the results of [24, Chapter VIII, Section 13]. For a fixed $z_{0}, w_{0} \in \mathbb{C}$, we let $\theta^{*}\left(t, z_{0}, w_{0}\right)$ be the function

$$
\theta^{*}\left(t, z_{0}, w_{0}\right)=\sum_{\gamma \in \Gamma}^{*} \exp \left(-t\left|z_{0}+\gamma\right|^{2} / A\right) \chi_{w_{0}}(\gamma)
$$

defined for $t>0$, where $\sum^{*}$ denotes the sum taken over all $\gamma \in \Gamma$ other than $-z_{0}$ if $z_{0}$ is in $\Gamma$. Furthermore, we let

$$
I\left(z_{0}, w_{0}, s\right):=\int_{1}^{\infty} \theta^{*}\left(t, z_{0}, w_{0}\right) t^{s-1} d t
$$

which converges for all $s \in \mathbb{C}$. Then by (31) in [24, Chapter VIII, Section 13] we have

$$
\begin{aligned}
A^{s} \Gamma(s) K_{0}^{*}\left(z_{0}, w_{0}, s\right)= & I\left(z_{0}, w_{0}, s\right)-\frac{\delta_{z_{0}}}{s} \chi_{z_{0}}\left(w_{0}\right) \\
& +I\left(w_{0}, z_{0}, 1-s\right) \chi_{z_{0}}\left(w_{0}\right)+\frac{\delta_{w_{0}}}{s-1},
\end{aligned}
$$

where $\delta_{x}=1$ if $x \in \Gamma$ and $\delta_{x}=0$ otherwise. The above integral expression gives the meromorphic continuation and the functional equation of $K_{0}^{*}\left(z_{0}, w_{0}, s\right)$. We now prove Theorem 1.1(i) using the second limit formula.

Proof of Theorem 1.1(i). From (5), we have

$$
\begin{aligned}
& A^{s-1} \Gamma(s)\left(A K_{0}^{*}(0,0, s)-\frac{1}{s-1}\right) \\
& \quad=I(0,0, s)-\frac{1}{s}+I(0,0,1-s)-\frac{A^{s-1} \Gamma(s)-1}{s-1} .
\end{aligned}
$$

Therefore, we have

(6) $\lim _{s \rightarrow 1}\left(A K_{0}^{*}(0,0, s)-\frac{1}{s-1}\right)=I(0,0,1)-1+I(0,0,0)-\log A+c$,

where $c$ is the Euler constant as before and we used the fact that $\Gamma^{\prime}(1)=-c$. On the other hand, we have for $z_{0}=0, w_{0}=z \notin \Gamma$, and $s=1$,

$$
A K_{0}^{*}(0, z, 1)=I(0, z, 1)-1+I(z, 0,0) .
$$

Note that $\lim _{z \rightarrow 0} I(0, z, 1)=I(0,0,1)$, and for $z \neq 0$, if we let

$$
I^{*}(z, 0, s)=I(z, 0, s)-\int_{1}^{\infty} \exp \left(-t|z|^{2} / A\right) t^{s-1} d t
$$


then $\lim _{z \rightarrow 0} I^{*}(z, 0,0)=I(0,0,0)$ by the definition of the sum $\sum^{*}$ in $(4)$. We have

$$
\begin{aligned}
\Gamma(s)-\frac{1}{s} & =\int_{|z|^{2} / A}^{\infty} e^{-t} t^{s-1} d t+\int_{0}^{|z|^{2} / A} e^{-t} t^{s-1} d t-\frac{1}{s} \\
& =\int_{|z|^{2} / A}^{\infty} e^{-t} t^{s-1} d t+\int_{0}^{|z|^{2} / A}\left(e^{-t}-1\right) t^{s-1} d t+\frac{1}{s}\left[\left(\frac{|z|^{2}}{A}\right)^{s}-1\right] .
\end{aligned}
$$

Taking $s \rightarrow 0$, we have

$$
-c=\int_{|z|^{2} / A}^{\infty} e^{-t} t^{-1} d t+\int_{0}^{|z|^{2} / A}\left(e^{-t}-1\right) t^{-1} d t+\log \left(\frac{|z|^{2}}{A}\right) .
$$

Hence

$$
\begin{aligned}
& A K_{0}^{*}(0, z, 1) \\
& \quad=I(0, z, 1)-1+I^{*}(z, 0,0)+\int_{1}^{\infty} \exp \left(-t|z|^{2} / A\right) t^{-1} d t \\
& \quad=I(0, z, 1)-1+I^{*}(z, 0,0)-c-\int_{0}^{|z|^{2} / A}\left(e^{-t}-1\right) t^{-1} d t-\log \left(\frac{|z|^{2}}{A}\right) .
\end{aligned}
$$

Therefore

$$
\lim _{z \rightarrow 0}\left(A K_{0}^{*}(0, z, 1)+\log |z|^{2}\right)=I(0,0,1)-1+I(0,0,0)-c+\log A .
$$

Finally, combining this with (6) and the second limit formula, we have

$$
\begin{aligned}
\lim _{s \rightarrow 1}\left(A K_{0}^{*}(0,0, s)-\frac{1}{s-1}\right) & =\lim _{z \rightarrow 0}\left(A K_{0}^{*}(0, z, 1)+\log |z|^{2}\right)-2 \log A+2 c \\
& =-\frac{1}{12} \log |\Delta|^{2}-2 \log A+2 c
\end{aligned}
$$

This proves our assertion.

\section{$\S 3$. Algebraic and $p$-adic properties of the Kronecker theta func- tion}

In this section, we first recall the definition and the generating function of Eisenstein-Kronecker numbers, and then we investigate the algebraic and $p$-adic properties of the function $F_{z_{0}, b}(z)$ defined in Definition 3.3. The $p$ adic Eisenstein-Kronecker functions will be defined in the next section as the iterated Coleman integrals of $F_{z_{0}, b}(z)$. 


\subsection{Eisenstein-Kronecker numbers and their generating func- tion}

We define the Eisenstein-Kronecker numbers to be the special values of Eisenstein-Kronecker-Lerch series (see also [5, Definition 1.5] and [7, Definition 2.3]).

Definition 3.1. Let $z_{0}, w_{0} \in \mathbb{C}$, and let $a$ and $b$ be integers such that $(a, b) \neq(-1,1)$ if $w_{0} \in \Gamma$. The Eisenstein-Kronecker number $e_{a, b}^{*}\left(z_{0}, w_{0}\right)$ is defined by $e_{a, b}^{*}\left(z_{0}, w_{0}\right):=K_{a+b}^{*}\left(z_{0}, w_{0}, b\right)$. As in [7, Definition 2.3], we let

$$
e_{a, b}^{*}\left(z_{0}\right):=e_{a, b}^{*}\left(0, z_{0}\right)=K_{a+b}^{*}\left(0, z_{0}, b\right)
$$

for $z_{0} \in \mathbb{C}$ such that $z_{0} \notin \Gamma$ if $(a, b) \neq(-1,1)$.

For any $z_{0}, w_{0} \in \mathbb{C}$, we let

$$
\Theta_{z_{0}, w_{0}}(z, w):=\exp \left[-\frac{z_{0} \bar{w}_{0}}{A}\right] \exp \left[-\frac{z \bar{w}_{0}+w \bar{z}_{0}}{A}\right] \Theta\left(z+z_{0}, w+w_{0}\right) .
$$

This function is known to be the generating function of Eisenstein-Kronecker numbers as follows (see [5, Section 1.14, Theorem 1.17]).

TheOREM 3.2. We have

$$
\Theta_{z_{0}, w_{0}}(z, w)=\chi_{z_{0}}\left(w_{0}\right) \frac{\delta_{z_{0}}}{z}+\frac{\delta_{w_{0}}}{w}+\sum_{a, b \geq 0}(-1)^{a+b} \frac{e_{a, b+1}^{*}\left(z_{0}, w_{0}\right)}{a ! A^{a}} z^{b} w^{a}
$$

in a neighborhood of the origin, where $\delta_{x}=1$ if $x \in \Gamma$ and $\delta_{x}=0$ otherwise.

We define the function $F_{z_{0}, b}(z)$ as in [7, Definition 4.2] as follows.

Definition 3.3. For any $z_{0} \in \mathbb{C}$ and integer $b \geq 0$, we define $F_{z_{0}, b}(z)$ to be the meromorphic function on $\mathbb{C}$ given by the equation

$$
\Theta_{z_{0}, 0}(z, w)=\sum_{b \geq 0} F_{z_{0}, b}(z) w^{b-1}
$$

The choice of $z_{0}$ in the definition of $F_{z_{0}, b}(z)$ depends only on the class of $z_{0}$ modulo $\Gamma$. When $z_{0}=0$, we let $F_{b}(z):=F_{0, b}(z)$. Explicit calculations show that we have $F_{0}(z)=1$ and $F_{1}(z)=\theta^{\prime}(z) / \theta(z)=\zeta(z)-e_{0,2}^{*} z$. By definition $\Theta_{z_{0}, 0}(z, w)=\exp \left(-w \bar{z}_{0} / A\right) \Theta\left(z+z_{0}, w\right)$. This equality and the definition of $F_{1}$ gives the equality $F_{z_{0}, 1}(z)=F_{1}\left(z+z_{0}\right)-\bar{z}_{0} / A$. We will later show that in the $p$-adic case, $F_{z_{0}, b}$ for various $z_{0}$ paste together to form a Coleman function.

The formula for $\Theta_{z_{0}, w_{0}}(z, w)$ as the generating function for EisensteinKronecker numbers gives the following. 
Proposition 3.4 (Generating function). For any $b \geq 0$, the Laurent expansion of $F_{z_{0}, b}(z)$ at zero is given by

$$
F_{z_{0}, b}(z)=\frac{\delta_{z_{0}, b}}{z}+\sum_{a \geq 0}(-1)^{a+b-1} \frac{e_{a, b}^{*}\left(z_{0}\right)}{a ! A^{a}} z^{a},
$$

where $\delta_{x, b}=1$ if $b=1$ and $x \in \Gamma$ and is zero otherwise.

Proof. The proposition follows from Theorem 3.2 and Definition 3.3.

Next, we assume that our complex torus has an algebraic model. Let $F$ be a number field with a fixed embedding $F \hookrightarrow \mathbb{C}$, and assume that we have an elliptic curve $E$ over $F$ defined by the Weierstrass equation

$$
E: y^{2}=4 x^{3}-g_{2} x-g_{3}
$$

We let $\Gamma$ be the period lattice of $E$ with respect to the invariant differential $\omega=d x / y$. We have a complex uniformization $\xi: \mathbb{C} / \Gamma \cong E(\mathbb{C})$ such that $d z$ corresponds to $\omega$.

We next define an auxiliary function $L_{n}(z)$, which is useful since it is an algebraic meromorphic function defined over $F$ (see Proposition 3.7).

Definition 3.5. Let $\Xi(z, w):=\exp \left(-F_{1}(z) w\right) \Theta(z, w)$. We define the connection function $L_{n}(z)$ by the formula

$$
\Xi(z, w):=\sum_{n \geq 0} L_{n}(z) w^{n-1} .
$$

REMARK 3.6. Explicit calculation shows that the connection function for small $n$ is given by $L_{0}(z)=1, L_{1}(z)=0$, and $L_{2}(z)=-\frac{1}{2} \wp(z)$.

The function $L_{n}(z)$ is a periodic with respect to $\Gamma$, hence defines a function on $\mathbb{C} / \Gamma$ holomorphic outside the points corresponding to $\Gamma$. The relation between $F_{z_{0}, b}(z)$ and $L_{n}(z)$ is given by the formula

$$
F_{z_{0}, b}(z)=\sum_{n=0}^{b} \frac{F_{z_{0}, 1}(z)^{b-n}}{(b-n) !} L_{n}\left(z+z_{0}\right) .
$$

The connection function $L_{n}(z)$ is algebraic in the following sense.

Proposition 3.7. The functions $L_{n}(z)$ correspond through the uniformization $\xi$ to rational functions $L_{n}$ on $E$ defined over $F$. 
Proof. See [7, Proposition 1.6] for the proof.

Assume now that $E$ has complex multiplication by the ring of integers $\mathcal{O}_{K}$ of an imaginary quadratic field $K$. In this case, the function $\Theta_{z_{0}, w_{0}}(z, w)$ satisfies the following algebraicity result.

Theorem 3.8 ([15, Theorem 1], [16, Theorem 2]). Suppose that $z_{0}, w_{0}$ correspond to torsion points in $\mathbb{C} / \Gamma \cong E(\mathbb{C})$. Then we have

$$
\Theta_{z_{0}, w_{0}}(z, w)-\chi_{z_{0}}\left(w_{0}\right) z^{-1} \delta_{z_{0}}-w^{-1} \delta_{w_{0}} \in \overline{\mathbb{Q}}[[z, w]],
$$

where $\delta_{x}=1$ if $x \in \Gamma$ and $\delta_{x}=0$ otherwise.

Proof. This is a reformulation of the classical theorem of Damerell. (See [5, Corollary 2.4] for a proof.)

Corollary 3.9. Suppose that $z_{0}$ corresponds to a torsion point in $\mathbb{C} / \Gamma \cong$ $E(\mathbb{C})$. Then we have

$$
F_{z_{0}, b}(z)-\delta_{z_{0}, b} z^{-1} \in \overline{\mathbb{Q}}[[z]]
$$

where $\delta_{x, b}=1$ if $b=1$ and $x \in \Gamma$, and $\delta_{x, b}=0$ otherwise.

We fix an isomorphism [ ] : $\mathcal{O}_{K} \cong \operatorname{End}_{\bar{F}}(E)$ so that $\alpha \in \mathcal{O}_{K}$ acts as $[\alpha]^{*} \omega=$ $\alpha \omega$ on the invariant differential $\omega=d x / y$. For any nonzero $\alpha \in \mathcal{O}_{K}$, we denote by $E[\alpha]$ the subgroup of $E(\overline{\mathbb{Q}})$ annihilated by $\alpha$. The function $F_{z_{0}, b}(z)$ is known to satisfy the following distribution relation with respect to $E[\alpha]$.

Proposition 3.10 (Distribution relation). The function $F_{z_{0}, b}(z)$ satisfies the relation

$$
\sum_{z_{\alpha} \in E[\alpha]} F_{z_{0}+z_{\alpha}, b}(z)=\alpha \bar{\alpha}^{1-b} F_{\alpha z_{0}, b}(\alpha z)
$$

for any nonzero $\alpha \in \mathcal{O}_{K}$.

Proof. By [5, Proposition 1.16], noting that

$$
\Theta_{\alpha z_{0}, 0}(\alpha z, w / \bar{\alpha} ; \Gamma)=\bar{\alpha} \Theta_{N(\alpha) z_{0}, 0}(N(\alpha) z, w ; \bar{\alpha} \Gamma),
$$

we see that the Kronecker theta function $\Theta_{z_{0}, 0}(z, w)$ satisfies the distribution relation

$$
\sum_{z_{\alpha} \in E[\alpha]} \Theta_{z_{0}+z_{\alpha}, 0}(z, w)=\alpha \Theta_{\alpha z_{0}, 0}(\alpha z, w / \bar{\alpha})
$$

for any nonzero $\alpha \in \mathcal{O}_{K}$. Our assertion follows from the definition of $F_{z_{0}, b}(z)$. 


\subsection{The $p$-adic properties of $F_{z_{0}, b}(z)$}

We next review the $p$-adic properties of the function $F_{z_{0}, b}(z)$. We let $E$ be an elliptic curve with complex multiplication by the ring of integers $\mathcal{O}_{K}$ of an imaginary quadratic field $K$. We assume in addition that $E$ is defined over $K$ and has good reduction at the primes above $p \geq 5$. This implies that $p$ does not ramify in $\mathcal{O}_{K}$. We fix a Weierstrass model of $E$ over $\mathcal{O}_{K}$ with good reduction above $p$, and by abuse of notation, we denote again by $E$ this model defined over $\mathcal{O}_{K}$. Let $t=-2 x / y$ be the formal parameter of $E$ at the origin, and denote by $\widehat{E}$ the formal group of $E$ with respect to the parameter $t$. We denote by $\lambda(t)$ the formal logarithm.

Let $z_{0}$ be a torsion point in $E(\overline{\mathbb{Q}})$, and denote by $\widehat{F}_{z_{0}, b}(t):=\left.F_{z_{0}, b}(z)\right|_{z=\lambda(t)}$ the formal composition of the Laurent expansion of $F_{z_{0}, b}(z)$ at the origin with the formal power series $z=\lambda(t)$. Note that by definition, we have

$$
\widehat{F}_{z_{0}, b}(t)=\sum_{n=0}^{b} \frac{\widehat{F}_{z_{0}, 1}(t)^{b-n}}{(b-n) !} \widehat{L}_{z_{0}, n}(t),
$$

where $\widehat{L}_{z_{0}, n}(t):=\left.L_{n}\left(z+z_{0}\right)\right|_{z=\lambda(t)}$.

Let $\psi:=\psi_{E / K}$ be the Hecke character of $K$ associated to $E$. We let $\mathfrak{p}$ be a prime in $\mathcal{O}_{K}$ lifting $p$, and we let $\pi:=\psi(\mathfrak{p})$. Note that if $p$ is ordinary, then $\pi$ is an element such that $p=\pi \pi^{*}$ with $\pi^{*}$ a unit in $K_{\mathfrak{p}}$, and $\pi=-p$ if $p$ is supersingular. We fix an embedding $\bar{K} \hookrightarrow \mathbb{C}_{p}$ such that $\pi$ maps to an element of $p$-adic absolute value less than 1 in $\mathbb{C}_{p}$. By Corollary 3.9, we may view $\widehat{F}_{z_{0}, b}(t)$ as a power series with coefficients in $\mathbb{C}_{p}$ through this embedding. We next review the $p$-adic properties of the power series $\widehat{F}_{z_{0}, b}(t)$ through this embedding.

Proposition 3.11. Let $z_{0}$ be a torsion point in $E(\overline{\mathbb{Q}})$ of order prime to $\mathfrak{p}$. Then the radius of convergence of the holomorphic part

$$
\widehat{F}_{z_{0}, b}(t)-\delta_{z_{0}, b} t^{-1} \in \mathbb{C}_{p}[[t]]
$$

of $\widehat{F}_{z_{0}, b}(t)$ is 1 . In other words, this power series defines a function on $\mathbb{B}^{-}:=\left\{\left.t \in \mathbb{C}_{p}|| t\right|_{p}<1\right\}$ if $b \neq 1$ or $z_{0} \neq 0$, and $\widehat{F}_{1}(t):=\widehat{F}_{0,1}(t)$ defines a function on $\mathbb{A}(0):=\left\{\left.t \in \mathbb{C}_{p}|0<| t\right|_{p}<1\right\}$.

Proof. See [7, Proposition 4.7] for the proof.

In the next section, we will prove that the power series $\widehat{F}_{z_{0}, b}(t)$ paste together to form a Coleman function on the elliptic curve minus the identity. 
We next review a formula for translation by $\pi^{n}$-torsion points. Let $\widehat{E}\left[\pi^{n}\right] \subset$ $\widehat{E}\left(\mathfrak{m}_{p}\right)$ denote the group of $\pi^{n}$-torsion points of the formal group $\widehat{E}$, where $\mathfrak{m}_{p}$ is the prime ideal of $\mathcal{O}_{\mathbb{C}_{p}}$.

LEMmA 3.12 (Translation). Suppose that $z_{0}$ is a torsion point in $E(\overline{\mathbb{Q}})$ of order prime to $\mathfrak{p}$. Let $t_{n} \in \widehat{E}\left[\pi^{n}\right]$, and let $z_{n}$ be the image of $t_{n}$ through the inclusion $\widehat{E}\left[\pi^{n}\right] \subset E(\overline{\mathbb{Q}}) \subset \mathbb{C} / \Gamma$. Then we have

$$
\widehat{F}_{z_{0}, b}\left(t \oplus t_{n}\right)=\widehat{F}_{z_{0}+z_{n}, b}(t),
$$

where $\oplus$ is the formal addition law of the formal group $\widehat{E}$.

Proof. See [7, Lemma 4.13] for the proof.

The above lemma gives the following corollary.

Corollary 3.13 (Generating function). Suppose that $z_{0}$ is a nonzero torsion point in $E(\overline{\mathbb{Q}})$ of order prime to $\mathfrak{p}$. Then for any integer $a \geq 0$, we have

$$
\left.\left(\partial_{t, \log }^{a} \widehat{F}_{z_{0}, b}(t)\right)\right|_{t=t_{n}}=(-1)^{a+b-1} e_{a, b}^{*}\left(z_{0}+z_{n}\right) / A^{a},
$$

where $\partial_{t, \log }$ is the differential operator $\lambda^{\prime}(t)^{-1} \partial_{t}$.

Proof. Since $\partial_{t, \log }$ is invariant under translation of the formal group, we have by Lemma 3.12

$$
\left.\left(\partial_{t, \log }^{a} \widehat{F}_{z_{0}, b}(t)\right)\right|_{t=t_{n}}=\left.\left(\partial_{t, \log }^{a} \widehat{F}_{z_{0}, b}\left(t \oplus t_{n}\right)\right)\right|_{t=0}=\left.\left(\partial_{t, \log }^{a} \widehat{F}_{z_{0}+z_{n}, b}(t)\right)\right|_{t=0} .
$$

Note that if we let $z=\lambda(t)$, then we have $\partial_{z}=\partial_{t, \log }$. Hence we have

$$
\left.\left(\partial_{t, \log }^{a} \widehat{F}_{z_{0}+z_{n}, b}(t)\right)\right|_{t=0}=\left.\left(\partial_{z}^{a} F_{z_{0}+z_{n}, b}(z)\right)\right|_{z=0} .
$$

Our assertion follows from the generating function property of $F_{z_{0}, b}$ given in Proposition 3.4.

We let $F_{1}^{(p)}(z)$ be the function

$$
F_{1}^{(p)}(z):=F_{1}(z)-\bar{\pi}^{-1} F_{1}(\pi z)
$$

which is an elliptic function corresponding to a rational function defined over $K$. Then we have $F_{1}^{(p)}\left(z+z_{0}\right)=F_{z_{0}, 1}(z)-\bar{\pi}^{-1} F_{\pi z_{0}, 1}(\pi z)$, and hence

$$
\left.F_{1}^{(p)}\left(z+z_{0}\right)\right|_{z=\lambda(t)}=\widehat{F}_{z_{0}, 1}(t)-\bar{\pi}^{-1} \widehat{F}_{\pi z_{0}, 1}([\pi] t) .
$$




\section{$\S 4$. Construction of the $p$-adic Eisenstein-Kronecker functions}

Coleman integration is a theory of $p$-adic integration first developed by Coleman [14] to define the $p$-adic polylogarithm function. In this section, we first review the theory of Coleman integration for curves, following the description of Besser [11], [12] using notation coming from the theory of rigid cohomology. We then prove that the function $F_{z_{0}, b}(z)$ of Section 3 defines a Coleman function $F_{b}^{\mathrm{col}}(z)$ on the CM elliptic curve. We then define the $p$-adic Eisenstein-Kronecker functions $E_{m, n}^{\mathrm{col}}(z)$ to be the iterated Coleman integral of $F_{b}^{\mathrm{col}}(z)$ satisfying the distribution relation.

\subsection{Review of Coleman integration}

Let $L$ be a complete subfield of $\mathbb{C}_{p}$, with ring of integers $\mathcal{O}_{L}$ and residue field $k$. Let $X$ be a smooth projective irreducible curve over $\mathcal{O}_{L}$. Let $U \subset X$ be an affine open subscheme of $X$ such that the complement $X \backslash U$ is a divisor flat over $\mathcal{O}_{L}$.

Denote by $X_{\mathbb{C}_{p}}^{\text {an }}$ the rigid analytic space associated to the scheme $X_{\mathbb{C}_{p}}$. Its points consist of the points of $X\left(\mathbb{C}_{p}\right)$. We have the specialization morphism

$$
\text { sp : } X_{\mathbb{C}_{p}}^{\mathrm{an}} \rightarrow X_{\overline{\mathbb{F}}_{p}},
$$

and we denote the inverse image of a point $x \in X\left(\overline{\mathbb{F}}_{p}\right)$ by $] x[$, which we call the residue disk. The set $] x\left[\subset X_{\mathbb{C}_{p}}^{\text {an }}\right.$ is an admissible open set of $X_{\mathbb{C}_{p}}^{\mathrm{an}}$. We have a set-theoretic decomposition

$$
\left.X_{\mathbb{C}_{p}}^{\mathrm{an}}=\coprod_{x \in X\left(\overline{\mathbb{F}}_{p}\right)}\right] x[
$$

which is not an admissible covering for the rigid topology.

We let $\mathcal{U}$ be the formal completion of $U$ with respect to the special fiber, we let $\mathcal{U}_{L}$ be the rigid analytic space over $L$ associated to the formal scheme $\mathcal{U}$, and we let $\mathcal{U}_{\mathbb{C}_{p}}:=\mathcal{U}_{L} \otimes_{L} \mathbb{C}_{p}$. We denote by $j: \mathcal{U}_{\mathbb{C}_{p}} \hookrightarrow X_{\mathbb{C}_{p}}^{\text {an }}$ the natural inclusion, and we let $j^{\dagger} \mathcal{O}_{\mathcal{U}_{\mathbb{C}_{p}}}$ be the ring of functions on $\mathcal{U}_{\mathbb{C}_{p}}$ overconvergent along $X_{\mathbb{C}_{p}}^{\text {an }} \backslash \mathcal{U}_{\mathbb{C}_{p}}$ (see [9, Section 2.1.1.3]).

Definition 4.1. For any $x \in X\left(\overline{\mathbb{F}}_{p}\right)$, we let $A(] x[)$ be the ring of functions defined by

$$
A(] x[):=\Gamma(] x\left[, j^{\dagger} \mathcal{O}_{\mathcal{U}_{\mathbb{C}_{p}}}\right) .
$$


Since $X$ is smooth, each residue disk is isomorphic to the open disk

$$
\mathbb{B}^{-}:=\left\{\left.t \in \mathbb{C}_{p}|| t\right|_{p}<1\right\}
$$

through a choice of local parameter $t_{x}$ of $X$ at $x$. Then we have

$$
\begin{aligned}
& A(] x[) \cong \Gamma\left(\mathbb{B}^{-}, \mathcal{O}_{\mathbb{B}^{-}}\right), \quad x \in U\left(\overline{\mathbb{F}}_{p}\right), \\
& A(] x[) \cong \bigcup_{0<r<1} \Gamma\left(\mathbb{A}(r), \mathcal{O}_{\mathbb{A}(r)}\right), \quad \text { otherwise, }
\end{aligned}
$$

where $\mathbb{A}(r)$ is defined to be the admissible open set of $\mathbb{B}^{-}$defined as the annulus $\mathbb{A}(r):=\left\{\left.t \in \mathbb{C}_{p}|r<| t\right|_{p}<1\right\}$ for any real number $r$ such that $0<$ $r<1$. Note that $A(] x[)$ is isomorphic to the ring consisting of formal power series $f\left(t_{x}\right)=\sum_{n \geq 0} a_{n} t_{x}^{n}$ which converge on $\mathbb{B}^{-}$if $x \in U\left(\overline{\mathbb{F}}_{p}\right)$, and formal power series $f\left(t_{x}\right)=\sum_{n=-\infty}^{\infty} a_{n} t_{x}^{n}$ for $a_{n} \in \mathbb{C}_{p}$ which converge on $\mathbb{A}(r)$ for some $r<1$ if $x \in(X \backslash U)\left(\overline{\mathbb{F}}_{p}\right)$. This description is independent of the choice of the parameter $t_{x}$.

Definition 4.2. A branch of the $p$-adic logarithm is any locally analytic group homomorphism $\log _{p}: \mathbb{C}_{p}^{\times} \rightarrow \mathbb{C}_{p}$, defined by the power series

$$
\log _{p}(x)=-\sum_{n>0} \frac{(1-x)^{n}}{n}
$$

for $x$ in a neighborhood of 1 . It is characterized by the value $\log _{p}(p)$.

Suppose a branch of the $p$-adic logarithm has been chosen. One defines $A_{\log }(] x[)$ to be $A(] x[)$ if $x \in U\left(\overline{\mathbb{F}}_{p}\right)$ and to be the polynomial ring in the function $\log _{p}\left(t_{x}\right)$ over $A(] x[)$ if $x \in(X \backslash U)\left(\overline{\mathbb{F}}_{p}\right)$. This definition is independent (up to isomorphism) of the choice of the local parameter $t_{x}$. Set $\Omega_{\log }^{1}(] x[):=A_{\log }(] x[) d t_{x}$. Then one defines the ring of locally analytic functions and 1-forms on $U$ by

$$
A_{\mathrm{loc}}(U):=\prod_{x \in X\left(\overline{\mathbb{F}}_{p}\right)} A_{\log }(] x[), \quad \Omega_{\mathrm{loc}}^{1}(U):=\prod_{x \in X\left(\overline{\mathbb{F}}_{p}\right)} \Omega_{\log }^{1}(] x[)
$$

We have a differential $d: A_{\text {loc }}(U) \rightarrow \Omega_{\text {loc }}^{1}(U)$, which is surjective. 
Suppose that $k=\mathbb{F}_{q}$ for $q=p^{h}$, and let $\mathcal{X}$ and $\mathcal{U}$ be the formal completions of $X$ and $U$ with respect to the special fiber. For simplicity, we assume that there exists a Frobenius morphism $\phi: \mathcal{X} \rightarrow \mathcal{X}$, which is a $\mathcal{O}_{K^{-}}$ linear morphism lifting the $h$ th power $\mathrm{Fr}^{h}$ of the absolute Frobenius Fr on $X_{k}:=X \otimes k$ and such that $\phi(\mathcal{U}) \subset \mathcal{U}$. Then this map induces a $\mathbb{C}_{p}$-morphism $\phi: X_{\mathbb{C}_{p}}^{\mathrm{an}} \rightarrow X_{\mathbb{C}_{p}}^{\mathrm{an}}$ by extension of scalars.

Coleman constructs a certain subring $M(U)$ of $A_{\text {loc }}(U)$, which we call the ring of Coleman functions on $U$, equipped with an integration map. The ring $M(U)$ is defined so that it contains rational functions on $X$ which are regular on $U$, as well as overconvergent functions on $\mathcal{U}_{\mathbb{C}_{p}} \subset X_{\mathbb{C}_{p}}^{\text {an }}$, where $\mathcal{U}_{\mathbb{C}_{p}}$ is the rigid analytic space associated to $\mathcal{U}$. If we denote by $M(U) / \mathbb{C}_{p}$ the ring $M(U)$ modulo addition by constants, then the integration map is a vector space map $\int: M(U) \otimes_{A(U)} \Omega^{1}(U) \rightarrow M(U) / \mathbb{C}_{p}$ characterized by the following three properties.

(i) We have $d\left(\int \omega\right)=\omega$ (primitive function).

(ii) We have $\int\left(\phi^{*} \omega\right) \equiv \phi^{*}\left(\int \omega\right)$ in $M(U) / \mathbb{C}_{p}$ (Frobenius invariance).

(iii) If $g \in M(U)$, then $\int d g \equiv g$ in $M(U) / \mathbb{C}_{p}$.

The construction of Coleman functions gives the following lemma.

Lemma 4.3. Suppose that $f$ is a function in $A_{\text {loc }}(U)$, and suppose that $P(x)$ is a polynomial in $\mathbb{C}_{p}$ whose roots do not contain the roots of unity. If we have $d f \in M(U) \otimes \Omega^{1}(U)$ and $P\left(\phi^{*}\right) f \in M(U)$, then we have $f \in M(U)$.

By abuse of notation, for any $\omega \in M(U) \otimes_{A(U)} \Omega^{1}(U)$, we denote by $\int \omega$ any Coleman function $F_{\omega} \in M(U)$ satisfying $d F_{\omega}=\omega$. By construction, the Coleman function $\int \omega \in M(U)$ is determined up to addition by a constant.

It is known that the above theory of integration is independent of the choice of the branch of the $p$-adic logarithm (see [19, Proposition 2.3]). Other important properties of Coleman functions are the uniqueness principle (see [14, Chapter IV] and [19, Proposition 2.4]) and the functorial property with respect to the morphisms of the pair $(U, X)$ (see [19, Proposition 2.5]).

\section{2. $F_{z_{0}, b}(z)$ as a Coleman function}

We will next show that the functions $F_{z_{0}, b}(z)$ defined in Section 3 define a Coleman function on our elliptic curve. It is striking that the functions modified for each $z_{0}$ nicely paste together to form a single Coleman function on the elliptic curve. We fix once and for all a branch of the $p$-adic logarithm.

Let the notation be as in Section 3.2. In particular, we let $E$ be the model over $\mathcal{O}_{K}$ of our $\mathrm{CM}$ elliptic curve, with good reduction at the primes 
above $p$. Let $L$ be a finite extension of $K_{\mathfrak{p}}$ in $\mathbb{C}_{p}$, and by abuse of notation, we denote again by $E$ the extension of $E$ to the ring of integers $\mathcal{O}_{L}$ of $L$. Then for $\pi:=\psi(\mathfrak{p})$, multiplication by $[\pi]$ induces a Frobenius $\phi: E \rightarrow E$. We denote by $E\left(\mathbb{C}_{p}\right):=E_{\mathbb{C}_{p}}^{\text {an }}$ the extension to $\mathbb{C}_{p}$ of the rigid analytic space $E_{L}^{\mathrm{an}}$. From now until the end of this paper, $z$ will denote a variable on $E\left(\mathbb{C}_{p}\right)$.

The residue disks of $E\left(\mathbb{C}_{p}\right)$ is parameterized by torsion points $z_{0}$ of $E$ of order prime to $\mathfrak{p}$. If we let $t=-2 x / y$, then this gives a local parameter of $E$ at the origin. Then the open disk $\left\{\left.t \in \mathbb{C}_{p}|| t\right|_{p}<1\right\}$ represents the residue disk $] 0\left[\subset E\left(\mathbb{C}_{p}\right)\right.$ containing the identity of $E$. If we let $z_{0}$ be a torsion point of $E$ of order prime to $\mathfrak{p}$, and if we let $] z_{0}\left[:=\tau_{z_{0}}(] 0[)\right.$ for the translation $\tau_{z_{0}}: E \rightarrow E$ defined by $\tau_{z_{0}}(z):=z+z_{0}$, then $] z_{0}[$ is precisely the residue disk containing $z_{0}$. The parameter $t$ of $] 0$ [ then gives via translation a parameter of $] z_{0}$ [ which gives a homeomorphism $] z_{0}\left[\cong \mathbb{B}^{-}\right.$between the residue disk containing $z_{0}$ and the unit ball. In what follows, we denote again by $t$ the parameter of $] z_{0}$ [ obtained in this fashion.

We first start by investigating the function $F_{z_{0}, 1}(z)$. We let $U:=E \backslash\{0\}$. Note that by Proposition 3.11, the power series $\widehat{F}_{z_{0}, 1}(t)$ defines a function in $A(] z_{0}[)$ through the identification $] z_{0}\left[\cong \mathbb{B}^{-}\right.$.

Lemma 4.4. We let $F_{1}^{\mathrm{col}}$ be the function in $A_{\mathrm{loc}}(U)$ defined by

$$
\left.F_{1}^{\mathrm{col}}\right|_{] z_{0}[}:=\widehat{F}_{z_{0}, 1}(t) \in A_{\log }(] z_{0}[)
$$

on each residue disk $] z_{0}\left[\right.$, where $z_{0}$ is a torsion point in $E(\overline{\mathbb{Q}})$ of order prime to $\mathfrak{p}$, including the case $z_{0}=0$. Then $F_{1}^{\text {col }}$ is a Coleman function on $U$.

Proof. The differential form $d F_{1}=\eta+e_{0,2}^{*} \omega$ is known to be a meromorphic differential form of the second kind on $U$ defined over $\mathcal{O}_{K}$. From the definition of $F_{z_{0}, 1}(z)$ given in Definition 3.3, we have $F_{z_{0}, 1}(z)=F_{1}\left(z+z_{0}\right)-\bar{z}_{0} / A$, hence $d F_{z_{0}, 1}=\tau_{z_{0}}^{*} d F_{1}$. Hence the calculation of the differential on each residue disk gives the equality $d F_{1}^{\mathrm{col}}=d F_{1}$. For any Coleman function $f(z)$ on $U$, denote by $f(\pi z)$ the Coleman function $\left(\phi^{*} f\right)(z)=[\pi]^{*} f(z)$. Consider the function

$$
\widetilde{F}_{1}^{(p)}(z):=\left(1-\frac{\phi^{*}}{\bar{\pi}}\right) F_{1}^{\mathrm{col}}(z)=F_{1}^{\mathrm{col}}(z)-\frac{1}{\bar{\pi}} F_{1}^{\mathrm{col}}(\pi z) .
$$

For any $z \in] z_{0}[$, we have $\widetilde{z}:=\pi z \in] \pi z_{0}[$. Hence from the definition and the fact that $[\pi] \circ \tau_{z_{0}}=\tau_{\pi z_{0}} \circ[\pi]$, we have

$$
\left.F_{1}^{\mathrm{col}}(\pi z)\right|_{] z_{0}[}=\left.F_{1}^{\mathrm{col}}(\widetilde{z})\right|_{] \pi z_{0}[}=\widehat{F}_{\pi z_{0}, 1}(\widetilde{t})=\widehat{F}_{\pi z_{0}, 1}([\pi] t),
$$


where $t$ and $\tilde{t}$ are elements in $\mathbb{B}^{-}$corresponding to $z$ and $\widetilde{z}$. Substituting the above equality into the definition $(12)$ of $\widetilde{F}_{1}^{(p)}(z)$, we obtain by (11) the equality

$$
\left.\widetilde{F}_{1}^{(p)}\right|_{] z_{0}[}=\left.F_{1}^{(p)}\right|_{] z_{0}[}
$$

where $F_{1}^{(p)}$ is the rational function on $E$ defined in (10). Hence $F_{1}^{\text {col }}$ is an element in $A_{\text {loc }}(U)$ such that $d F_{1}^{\mathrm{col}}=d F_{1}$ and $\left(1-\phi^{*} / \bar{\pi}\right) F_{1}^{\mathrm{col}}=F_{1}^{(p)}$. Since $F_{1}^{(p)}$ is a rational function and $d F_{1}$ is a meromorphic differential form, which are both regular on $U$, we have in particular $F_{1}^{(p)} \in M(U)$ and $d F_{1} \in$ $M(U) \otimes \Omega^{1}(U)$. Hence by Lemma 4.3 , the function $F_{1}^{\text {col }}$ is in fact a Coleman function on $U$.

Note that if $z_{0} \neq 0$, then we have $\left.F_{1}^{\mathrm{col}}\right|_{] z_{0}[} \in A(] z_{0}[) \subset A_{\log }(] z_{0}[)$. This shows that the value $F_{1}^{\mathrm{col}}(z)$ for $\left.z \in E\left(\mathbb{C}_{p}\right) \backslash\right] 0[$ is independent of the choice of the branch of the $p$-adic logarithm.

Since $L_{n}$ is a rational function on $E$ with poles only at $\{0\}$ in $E$, it is in particular a Coleman function on $U$. The set of Coleman functions is a ring, and we define $F_{b}^{\text {col }}$ as follows.

Definition 4.5. We let $F_{1}^{\text {col }}$ be the Coleman function of Lemma 4.4. For any integer $b \geq 0$, we define $F_{b}^{\text {col }}$ to be the Coleman function

$$
F_{b}^{\mathrm{col}}:=\sum_{n=0}^{b} \frac{\left(F_{1}^{\mathrm{col}}\right)^{b-n}}{(b-n) !} L_{n}
$$

on $U$.

The functions $F_{b}^{\mathrm{col}}$ interpolate the power series $F_{z_{0}, b}(z)$ of Section 4.1.

Proposition 4.6 (Interpolation). For any integer $b \geq 0$, the function $F_{b}^{\mathrm{col}}$ on the residue disk $] z_{0}[$ is given by

$$
\left.F_{b}^{\mathrm{col}}\right|_{z_{0}[}=\widehat{F}_{z_{0}, b}(t) \in A_{\log }(] z_{0}[) .
$$

Proof. The case for $b=1$ follows from the definition of $F_{1}^{\mathrm{col}}$. The case for $b>1$ follows from this case, noting that $\left.L_{n}\right|_{z_{0}[}=\widehat{L}_{z_{0}, n}(t)$ and comparing the definitions of (9) and (13).

Proposition 4.7 (Distribution relation). For any nonzero $\alpha \in \mathcal{O}_{K}$, the Coleman function $F_{b}^{\mathrm{col}}$ for any integer $b \geq 0$ satisfies the distribution relation

$$
\sum_{z_{\alpha} \in E[\alpha]} F_{b}^{\mathrm{col}}\left(z+z_{\alpha}\right)=\alpha \bar{\alpha}^{1-b} F_{b}^{\mathrm{col}}(\alpha z) .
$$


Note that since $F_{b}^{\mathrm{col}}$ is a Coleman function on $U$, by the functorial property of Coleman functions (see [19, Proposition 2.5]), we may regard both $F_{b}^{\mathrm{col}}\left(z+z_{\alpha}\right)$ and $F_{b}^{\mathrm{col}}(\alpha z)$ as Coleman functions on $U_{\alpha}:=E \backslash E[\alpha]$. Then (14) above is an equality of Coleman functions on $U_{\alpha}$.

Proof of Proposition 4.7. We write $\alpha=\pi^{n} \alpha_{0}$, where $\alpha_{0} \in \mathcal{O}_{K}$ is prime to $\pi$. For any $z_{\alpha} \in E[\alpha]$, we write $z_{\alpha}=z_{\alpha_{0}}+z_{n}$, where $z_{\alpha_{0}} \in E\left[\alpha_{0}\right]$ and $z_{n} \in E\left[\pi^{n}\right]$. Suppose that $z$ is a point in the residue disk $] z_{0}\left[\right.$. Then $z+z_{\alpha}$ is in the residue disk $] z_{0}+z_{\alpha_{0}}[$, and $\alpha z$ is in $] \alpha z_{0}[$. Denote by $t$ the parameter on $] z_{0}$ [ obtained as the translation by $\tau_{z_{0}}: E \rightarrow E$ of the local parameter $t=-2 x / y$ at the origin of $E$. This gives an isomorphism $] z_{0}\left[\cong \mathbb{B}^{-}\right.$, and hence $t$ parameterizes the points $z$ on the residue disk $] z_{0}[$. We denote by $t_{n}$ the element on $\mathbb{B}^{-}$corresponding to $z_{n}$ through this isomorphism. Then Proposition 4.6 shows that we have

$$
\begin{aligned}
\left.F_{b}^{\mathrm{col}}(\alpha z)\right|_{] z_{0}[} & =\widehat{F}_{\alpha z_{0}, b}([\alpha] t), \\
\left.F_{b}^{\mathrm{col}}\left(z+z_{\alpha}\right)\right|_{] z_{0}[} & =\widehat{F}_{z_{0}+z_{\alpha_{0}}, b}\left(t \oplus t_{n}\right)
\end{aligned}
$$

as functions on $] z_{0}\left[\right.$. By Lemma 3.12, we have $\widehat{F}_{z_{0}+z_{\alpha_{0}}, b}\left(t \oplus t_{n}\right)=\widehat{F}_{z_{0}+z_{\alpha}, b}(t)$. Our result now follows by substituting $z=\lambda(t)$ into the distribution relation of Proposition 3.10, noting that $\left.F_{z_{0}, b}(\alpha z)\right|_{z=\lambda(t)}=\left.F_{z_{0}, b}(z)\right|_{z=\lambda([\alpha] t)}$.

REMARK 4.8 .

(i) The function $F_{1}^{\mathrm{col}}$ is characterized as the unique function of the form $F_{1}^{\mathrm{col}}=\int d F_{1}$ satisfying the distribution relation.

(ii) The convergence property for $\widehat{F}_{z_{0}, 1}(t)$ shows that the function $F_{1}^{\text {col }}$ converges on any point in $U\left(\mathbb{C}_{p}\right)$.

(iii) Furthermore, the expansion of $\widehat{F}_{b}^{\text {col }}(t)$ for $b>1$ shows that $F_{b}^{\text {col }}$ is defined on $E\left(\mathbb{C}_{p}\right)$.

\subsection{The $p$-adic Eisenstein-Kronecker function}

We now define the $p$-adic Eisenstein-Kronecker function. We first prepare a proposition concerning integration and the distribution relation.

Proposition 4.9. Let $m$ and $b$ be integers greater than or equal to zero. Suppose that $E_{m, b}^{\mathrm{col}}$ is a Coleman function on $U$ which satisfies the distribution relation

$$
\sum_{z_{\alpha} \in E[\alpha]} E_{m, b}^{\mathrm{col}}\left(z+z_{\alpha}\right)=\alpha^{1-m} \bar{\alpha}^{1-b} E_{m, b}^{\mathrm{col}}(\alpha z)
$$


for any nonzero $\alpha \in \mathcal{O}_{K}$. Then there exists a unique integration $E_{m+1, b}^{\mathrm{col}}:=$ $-\int E_{m, b}^{\mathrm{col}} \omega$ of $-E_{m, b}^{\mathrm{col}} \omega$ satisfying the distribution relation

$$
\sum_{z_{\alpha} \in E[\alpha]} E_{m+1, b}^{\mathrm{col}}\left(z+z_{\alpha}\right)=\alpha^{-m} \bar{\alpha}^{1-b} E_{m+1, b}^{\mathrm{col}}(\alpha z)
$$

for any nonzero $\alpha \in \mathcal{O}_{K}$.

Proof. Let $\widetilde{E}_{m+1, b}:=-\int E_{m, b}^{\mathrm{col}} \omega$ be any Coleman integral of $-E_{m, b}^{\mathrm{col}} \omega$. For any nonzero $\alpha \in \mathcal{O}_{K}$, let

$$
c_{\alpha}:=\sum_{z_{\alpha} \in E[\alpha]} \widetilde{E}_{m+1, b}\left(z+z_{\alpha}\right)-\alpha^{-m} \bar{\alpha}^{1-b} \widetilde{E}_{m+1, b}(\alpha z) .
$$

Then the relation (15) shows that $d c_{\alpha}=0$, and hence the property of Coleman integration shows that $c_{\alpha}$ is a constant in $\mathbb{C}_{p}$. For any nonzero $\alpha$, $\beta \in \mathcal{O}_{K}$, we have

$$
\begin{aligned}
& \sum_{z_{\alpha \beta} \in E[\alpha \beta]} \widetilde{E}_{m+1, b}\left(z+z_{\alpha \beta}\right) \\
& =\sum_{\substack{z_{\alpha} \in E[\alpha], \widetilde{z}_{\beta} \in E[\alpha \beta] / E[\alpha]}} \widetilde{E}_{m+1, b}\left(z+z_{\alpha}+\widetilde{z}_{\beta}\right) \\
& =\sum_{\widetilde{z}_{\beta} \in E[\alpha \beta] / E[\alpha]}\left(c_{\alpha}+\alpha^{-m} \bar{\alpha}^{1-b} \widetilde{E}_{m+1, b}\left(\alpha z+\alpha \widetilde{z}_{\beta}\right)\right) \\
& =N(\beta) c_{\alpha}+\alpha^{-m} \bar{\alpha}^{1-b} c_{\beta}+(\alpha \beta)^{-m}(\overline{\alpha \beta})^{1-b} \widetilde{E}_{m+1, b}(\alpha \beta z),
\end{aligned}
$$

where the last equality follows from the definition of $c_{\beta}$ and the fact that we have an isomorphism $E[\alpha \beta] / E[\alpha] \cong E[\beta]$ given by $\widetilde{z}_{\beta} \mapsto z_{\beta}:=\alpha \widetilde{z}_{\beta}$. By reversing the roles of $\alpha$ and $\beta$, we see from a similar calculation that the above is also equal to

$$
N(\alpha) c_{\beta}+\beta^{-m} \bar{\beta}^{1-b} c_{\alpha}+(\alpha \beta)^{-m}(\overline{\alpha \beta})^{1-b} \widetilde{E}_{m+1, b}(\alpha \beta z) .
$$

This shows that we have $\left(N(\beta)-\beta^{-m} \bar{\beta}^{1-b}\right) c_{\alpha}=\left(N(\alpha)-\alpha^{-m} \bar{\alpha}^{1-b}\right) c_{\beta}$, and hence the constant

$$
c:=c_{\alpha} /\left(N(\alpha)-\alpha^{-m} \bar{\alpha}^{1-b}\right)
$$

is independent of the choice of $\alpha \in \mathcal{O}_{K}$. If we let

$$
E_{m+1, b}^{\mathrm{col}}(z):=\widetilde{E}_{m+1, b}(z)-c
$$

then this function satisfies (16) for any nonzero $\alpha \in \mathcal{O}_{K}$. 
Definition 4.10. For integers $m, b$ with $b \geq 0$, we define the $p$-adic Eisenstein-Kronecker series $E_{m, b}^{\mathrm{col}}$ recursively going up and down as follows. We first let $E_{0, b}^{\mathrm{col}}:=(-1)^{b-1} F_{b}^{\mathrm{col}}$. Then by Proposition 4.7 , this function satisfies the distribution relation (15). For $m>0$, we let $E_{m, b}^{\mathrm{col}}$ be the Coleman function recursively defined by $E_{m, b}^{\mathrm{col}}=-\int E_{m-1, b}^{\mathrm{col}} \omega$, with the constant term normalized as in Proposition 4.9 to satisfy the distribution relation

$$
\sum_{z_{\alpha} \in E[\alpha]} E_{m, b}^{\mathrm{col}}\left(z+z_{\alpha}\right)=\alpha^{1-m} \bar{\alpha}^{1-b} E_{m, b}^{\mathrm{col}}(\alpha z) .
$$

For $m<0$, we define $E_{m, b}^{\mathrm{col}}$ recursively by the formula $d E_{m+1, b}^{\mathrm{col}}=-E_{m, b}^{\mathrm{col}} \omega$.

Again, (17) is an equality of Coleman functions on $U_{\alpha}:=E \backslash E[\alpha]$. Proposition 4.9 ensures that such a choice of constant term when $m>0$ is possible. The convergence property of $F_{1}$ in Proposition 3.11 ensures that $E_{m, b}^{\mathrm{col}}$ is defined on any point in $U\left(\mathbb{C}_{p}\right)$ if $b=1$ and on $E\left(\mathbb{C}_{p}\right)$ if $b>1$. When $b=0$, note that $E_{0,0}^{\text {col }} \equiv 1$. This shows that we have $E_{a, 0}^{\text {col }}=0$ for $a<0$.

The reason we view $E_{m, b}^{\mathrm{col}}$ as a $p$-adic analogue of the Eisenstein-Kronecker function is that this function interpolates values of the classical EisensteinKronecker function at torsion points for $m \leq 0$ as follows.

Proposition 4.11. Let $a, b$ be integers greater than or equal to zero. Then for any torsion point $z$ in $E(\overline{\mathbb{Q}})$ such that $z \neq 0$ if $b=1$, we have

$$
E_{-a, b}^{\mathrm{col}}(z)=E_{-a, b}(z) / A^{a}
$$

Proof. Any torsion point $z$ is of the form $z=z_{0}+z_{n}$, where $z_{0}$ is a Teichmüller representative and $z_{n}$ is a $\pi^{n}$-torsion point. Our result follows from the fact that

$$
E_{-a, b}^{\mathrm{col}}(z)=\left.(-1)^{a+b-1} \partial_{t, \log }^{a} \widehat{F}_{z_{0}, b}(t)\right|_{t=t_{n}}
$$

and Corollary 3.13.

Note that we have fixed a branch of the $p$-adic logarithm. We next prove that the values of $E_{m, b}^{\mathrm{col}}(z)$ are independent of this choice.

LEMMA 4.12. Let $m$ and $b$ be integers such that $b \geq 0$. Suppose that $z$ is a point in $\left.E\left(\mathbb{C}_{p}\right) \backslash\right] 0\left[\right.$. Then the value $E_{m, b}^{\mathrm{col}}(z)$ is independent of the choice of the branch of the p-adic logarithm. 
Proof. The proof is given by induction on $m$. The statement for $m=0$ and $b=1$ follows from the construction of $F_{1}^{\mathrm{col}}$, which is independent of the choice of the branch of the $p$-adic logarithm. The statement for $m=0$ and general $b$ follows from the definition (13) of $F_{b}^{\text {col }}$, noting that $L_{n}$ are rational functions. The statement for $m<0$ follows inductively from the equality $E_{m, b}^{\mathrm{col}} \omega=-d E_{m+1, b}^{\mathrm{col}}$. Suppose now that the statement is true for some $m \geq 0$. Let $\widetilde{E}_{m+1, b}=-\int E_{m, b} \omega$ be any Coleman integral. By Besser's formalism (see [12]), Coleman integrals over points of good reduction (i.e., points in the smooth subscheme $U \subset X$ using the notation of Section 4.1) are free from the choice of the branch of the $p$-adic logarithm. Hence for $z$ in $\left.E\left(\mathbb{C}_{p}\right) \backslash\right] 0\left[\right.$, the value $\widetilde{E}_{m+1, b}(z)$ is independent of the choice of the branch. Furthermore, for a point in $z$ in $\left.E\left(\mathbb{C}_{p}\right) \backslash\right] 0\left[\right.$, the values $\widetilde{E}_{m+1, b}(\pi z)$ and $\widetilde{E}_{m+1, b}\left(z+z_{1}\right)$ for $z_{1} \in E[\pi]$ are also free from the choice of the branch. Hence the global constant $c=c_{\pi}$ of Proposition 4.9 is independent of the choice of the branch. This gives the statement for $E_{m+1, b}^{\mathrm{col}}(z)$.

Remark 4.13. The restriction of $E_{m, b}^{\mathrm{col}}(z)$ to the residue disk $] 0[$ is of the form

$$
\left.E_{m, b}^{\mathrm{col}}\right|_{0[0}=\widehat{a}_{0}(t)+\widehat{a}_{1}(t) \log t+\widehat{a}_{2}(t)(\log t)^{2}+\cdots+\widehat{a}_{n}(t)(\log t)^{n}
$$

for some $n$ and $\widehat{a}_{i}(t) \in A(] 0[)$, where the $\widehat{a}_{i}(t)$ are rigid analytic functions on an open annulus around zero free from the choice of the branch. Since $E_{m, b}^{\text {col }}(z)$ for $b \neq 1$ is analytic on $E\left(\mathbb{C}_{p}\right)$, we see that $n=0$ in this case. Therefore if $b \neq 1$, then the values $E_{m, b}^{\text {col }}(z)$ for $z \in E\left(\mathbb{C}_{p}\right)$ are independent of the choice of the branch. On the other hand, if $b=1$ and $m>0$, then $E_{m, b}^{\mathrm{col}}(z)$ for $z$ in the residue disk $] 0[$ depends on the choice of the branch of the $p$-adic logarithm.

\section{$\S 5$. $p$-adic Kronecker limit formulas}

Let the notation be as in Section 4. In this section, we prove the $p$-adic analogues of the first and second Kronecker limit formulas.

\subsection{The $p$-adic Eisenstein-Kronecker functions}

In Section 4, we defined the $p$-adic analogue of the Eisenstein-Kronecker series as a Coleman function on the CM elliptic curve. In order to prove the $p$-adic limit formulas, we define in this section a $p$-adic analogue of the function $\log |\theta(z)|^{2}-|z|^{2} / A$, which turns out to be a Coleman function. 
We then prove the distribution relation, which we use to characterize this function.

Let $p$ be a prime greater than or equal to 5 . In what follows, fix an embedding of $\overline{\mathbb{Q}}$ into $\mathbb{C}_{p}$, and we again fix a branch of the $p$-adic logarithm, which is a homomorphism $\log _{p}: \mathbb{C}_{p}^{\times} \rightarrow \mathbb{C}_{p}$, and we extend this homomorphism to $\left(\mathbb{C}_{p}[[t]]\right)^{\times}$by using the decomposition $\left(\mathbb{C}_{p}[[t]]\right)^{\times}=\mathbb{C}_{p}^{\times} \times\left(1+t \mathbb{C}_{p}[[t]]\right)$ and defining $\log _{p}(1-t f(t))=-\sum t^{n} f^{n}(t) / n$ for any $f(t) \in \mathbb{C}_{p}[[t]]$. Let $E$ be a $\mathrm{CM}$ elliptic curve as in the Introduction, and let $\Gamma$ be the period lattice of $E \otimes \mathbb{C}$. For $z_{0} \in \Gamma \otimes \mathbb{Q}$, we let

$$
\theta_{z_{0}}(z):=\theta\left(z+z_{0}\right) \exp \left(-\frac{z \bar{z}_{0}}{A}-\frac{z_{0} \bar{z}_{0}}{2 A}\right)
$$

Then by [5, Proposition 2.1], the Taylor series of $\theta_{z_{0}}(z)$ at $z=0$ has algebraic coefficients. Note that from the definition, we have

$$
\frac{d}{d z} \log \theta_{z_{0}}(z)=\frac{\theta_{z_{0}}^{\prime}(z)}{\theta_{z_{0}}(z)}=\frac{\theta^{\prime}\left(z+z_{0}\right)}{\theta\left(z+z_{0}\right)}-\frac{\bar{z}_{0}}{A}=F_{1}\left(z+z_{0}\right)-\frac{\bar{z}_{0}}{A}=F_{z_{0}, 1}(z) .
$$

If we consider the formal composition

$$
\widehat{\theta}_{z_{0}}(t):=\left.\theta_{z_{0}}(z)\right|_{z=\lambda(t)}
$$

of this series with $\lambda(t)$, where $\lambda(t)$ is the formal logarithm of the formal group of $E$, then we may regard this power series as an element in $\mathbb{C}_{p}[[t]]$. If we take the derivative of $\log _{p} \widehat{\theta}_{z_{0}}(t)$ with respect to $t$, then we have by the definition of $\log _{p}$ and $\widehat{\theta}_{z_{0}}(t)$ the equality

$$
\left(\log _{p} \widehat{\theta}_{z_{0}}(t)\right)^{\prime}=\widehat{\theta}_{z_{0}}^{\prime}(t) / \widehat{\theta}_{z_{0}}(t)=\widehat{F}_{z_{0}, 1}(t) .
$$

If $z_{0}$ corresponds to a torsion point of $E$ of order prime to $\mathfrak{p}$, then we see from Proposition 3.11 that the power series $\left(\log _{p} \widehat{\theta}_{z_{0}}(t)\right)^{\prime}$, hence also $\log _{p} \widehat{\theta}_{z_{0}}(t)$, converges on the open unit disk $|t|<1$ in $\mathbb{C}_{p}$.

Definition 5.1. We let $\log _{p} \theta$ be the function in $A_{\text {loc }}(U)$ defined by

$$
\left.\log _{p} \theta\right|_{] z_{0}[}:=\log _{p} \widehat{\theta}_{z_{0}}(t) \in A_{\log }(] z_{0}[)
$$

on each residue disk $] z_{0}\left[\right.$, where $z_{0}$ corresponds to a torsion point of $E$ of order prime to $\mathfrak{p}$.

We will see in Section 5.2 that $\log _{p} \theta$ is in fact a Coleman function on $E$. We first investigate the basic properties of $\log _{p} \theta$. 
Proposition 5.2. For $z_{0} \in \Gamma \otimes \mathbb{Q}$ and $z_{\alpha}$ such that $\alpha z_{\alpha} \in \Gamma$ for a $\pi$-power morphism $\alpha \in \operatorname{End}_{\overline{\mathbb{Q}}}(E)$, we have

$$
\log _{p} \widehat{\theta}_{z_{0}}\left(t \oplus t_{\alpha}\right)=\log _{p} \widehat{\theta}_{z_{0}+z_{\alpha}}(t)
$$

where $t_{\alpha} \in E\left(\mathbb{C}_{p}\right)$ corresponds to a torsion point in $\mathbb{C} / \Gamma$ represented by $z_{\alpha} \in$ $\mathbb{C}$.

Proof. Let $\alpha$ and $\beta$ be elements of $\mathcal{O}_{K}$ such that $2 \alpha \mid \beta$ and $\beta z_{0} \in \Gamma$. Then $f_{\beta}(z):=\theta(z)^{N \beta} / \theta(\beta z)$ is a rational function on $E$ over $\overline{\mathbb{Q}}$. We have

$$
\theta_{z_{0}+z_{\alpha}}(z)^{N \beta}= \pm \theta(\beta z) \tau_{z_{0}+z_{\alpha}}^{*} f_{\beta}(z)
$$

Similarly, we have

$$
\theta_{z_{0}}(z)^{N \beta}= \pm \theta(\beta z) \tau_{z_{0}}^{*} f_{\beta}(z)
$$

Since $f_{\beta}$ is a rational function, we also have

$$
\tau_{z_{0}+z_{\alpha}}^{*} f_{\beta}(t)=\tau_{z_{0}}^{*} f_{\beta}\left(t \oplus t_{\alpha}\right)
$$

Hence we have

$$
\widehat{\theta}_{z_{0}}\left(t \oplus t_{\alpha}\right)^{N \beta}= \pm \widehat{\theta}([\beta] t) \tau_{z_{0}+z_{\alpha}}^{*} f_{\beta}(t)
$$

Our assertion now follows from (21) and (22).

Corollary 5.3. Let $t_{\alpha}$ be a $\pi$-power torsion point, and assume that $z_{0} \neq 0$ or $t_{\alpha} \neq 0$. Then we have

$$
\log _{p} \widehat{\theta}_{z_{0}}\left(t_{\alpha}\right)=\log _{p}\left(\theta\left(z_{0}+z_{\alpha}\right) \exp \left[-\frac{\left(z_{0}+z_{\alpha}\right) \overline{\left(z_{0}+z_{\alpha}\right)}}{2 A}\right]\right) .
$$

Proof. This follows by substituting $t=0$ on both sides of (20) and using the definition of $\theta_{z_{0}+z_{\alpha}}(z)$ given in (18).

Roughly speaking, $\log _{p} \theta(z)$ is a $p$-adic function which interpolates the special values $\log \theta(z)-z \bar{z} / 2 A$ at torsion points. We may thus regard $\log _{p} \theta(z)$ as a $p$-adic analogue of the function $\log |\theta(z)|^{2}-|z|^{2} / A$. 


\subsection{The $p$-adic second limit formula}

We are now ready to prove Theorem 1.2 , which is a $p$-adic analogue of the Kronecker second limit formula. We keep the notation of the Introduction. In particular, let

$$
K_{n-m}^{\mathrm{col}}(0, z, n):=E_{m, n}^{\mathrm{col}}(z) .
$$

In addition, we let $\mathfrak{f} \subset \mathcal{O}_{K}$ be the conductor of the Hecke character $\psi$ of $K$ associated to $E$, and we let $\pi:=\psi(\mathfrak{p})$. Since $E$ has good reduction at the prime ideals above $p$, we have $(p, \mathfrak{f})=1$.

Proposition 5.4. Let $z_{0} \in \mathbb{C}$ be a lifting of an $\mathfrak{f}$-torsion point of $\mathbb{C} / \Gamma$. Then for $\alpha \in \mathcal{O}_{K}$, we have

$$
\theta_{\alpha z_{0}}(\alpha z)^{24 N(\alpha \mathfrak{f})}=\Delta^{2 N(\alpha \mathfrak{f})(N \alpha-1)} \prod_{z_{\alpha} \in E[\alpha]} \theta_{z_{0}+z_{\alpha}}(z)^{24 N(\alpha \mathfrak{f})},
$$

where $z_{\alpha}$ is a lift of an $\alpha$-torsion point of $E$, and the right-hand side is independent of the choice of the lifts $z_{0}$ and $z_{\alpha}$ on $\mathbb{C}$.

Proof. Since for $\gamma \in \Gamma$ we have $\theta_{z_{0}+\gamma}(z)= \pm \chi_{\gamma}\left(z_{0} / 2\right) \theta_{z_{0}}(z)$, the function $\theta_{z_{0}}(z)^{2 N \mathfrak{f}}$ is independent of the lift $z_{0}$ if $(N \mathfrak{f}) z_{0} \in \Gamma$. The independence of the lifts of $z_{0}$ and $z_{\alpha}$ follows from this fact. The logarithmic derivatives of both sides coincide by Proposition 3.10. Hence for each $\alpha$, there exists a constant $c_{\alpha}\left(z_{0}\right)$ such that

$$
\theta_{\alpha z_{0}}(\alpha z)^{2 N(\alpha \mathfrak{f})}=c_{\alpha}\left(z_{0}\right) \prod_{z_{\alpha} \in E[\alpha]} \theta_{z_{0}+z_{\alpha}}(z)^{2 N(\alpha \mathfrak{f})} .
$$

If we compare this equality with the case $z_{0}=0$, then we have from the definition of $\theta_{z_{0}}(z)$ given in (18) the equality

$$
c_{\alpha}(0)=c_{\alpha}\left(z_{0}\right) \prod_{z_{\alpha}} \exp \left(\frac{z_{0} \bar{z}_{\alpha}-\bar{z}_{0} z_{\alpha}}{2 A}\right)^{2 N(\alpha \mathfrak{f})}=c_{\alpha}\left(z_{0}\right) .
$$

Hence we see that $c_{\alpha}:=c_{\alpha}\left(z_{0}\right)$ is independent of the choice of $z_{0}$. We calculate $c_{\alpha}$ for the case $z_{0}=0$ and $N \mathfrak{f}=1$. Then we have

$$
\begin{aligned}
& \prod_{z_{\alpha \beta} \in E[\alpha \beta]} \theta_{z_{\alpha \beta}(z)^{2 N(\alpha \beta)}} \\
& =\prod_{z_{\alpha \beta} \in E[\alpha \beta] / E[\alpha]} \prod_{z_{\alpha} \in E[\alpha]} \theta_{z_{\alpha \beta}+z_{\alpha}}(z)^{2 N(\alpha \beta)}
\end{aligned}
$$




$$
\begin{aligned}
& =\prod_{z_{\alpha \beta} \in E[\alpha \beta] / E[\alpha]} c_{\alpha}^{-N \beta} \theta_{\alpha z_{\alpha \beta}}(\alpha z)^{2 N(\alpha \beta)} \\
& =c_{\alpha}^{-N \beta^{2}} c_{\beta}^{-N \alpha} \theta(\beta \alpha z)^{2 N(\alpha \beta)} .
\end{aligned}
$$

Hence we have $c_{\alpha}^{N \beta^{2}} c_{\beta}^{N \alpha}=c_{\alpha \beta}=c_{\beta}^{N \alpha^{2}} c_{\alpha}^{N \beta}$, or equivalently,

$$
c_{\alpha}^{N \beta(N \beta-1)}=c_{\beta}^{N \alpha(N \alpha-1)} .
$$

In particular, $c_{\alpha}^{12}=c_{2}^{N \alpha(N \alpha-1)}$. On the other hand, we consider the constant term of

$$
\frac{\theta(2 z)^{8}}{\theta(z)^{8}}=c_{2} \prod_{z_{2} \in E[2]-\{0\}} \theta_{z_{2}}(z)^{8}
$$

As in the proof of Proposition 2.8, we have

$$
\prod_{z_{2} \in E[2]-\{0\}} \theta_{z_{2}}(0)^{8}=\Delta^{\prime-2}
$$

Hence $c_{2}=2^{8} \Delta^{\prime 2}=\Delta^{2}$. Our assertion now follows from these facts.

Corollary 5.5. The function $\Xi(z):=-\log _{p} \theta(z)-\frac{1}{12} \log _{p} \Delta$ satisfies the distribution relation

$$
\Xi(\alpha z)=\sum_{z_{\alpha} \in E[\alpha]} \Xi\left(z+z_{\alpha}\right) .
$$

Proof. By Proposition 5.4, we have

$$
\log _{p} \widehat{\theta}_{\alpha z_{0}}([\alpha] t)=\frac{N \alpha-1}{12} \log _{p} \Delta+\sum_{t_{\alpha} \in E[\alpha]} \log _{p} \widehat{\theta}_{z_{0}}\left(t \oplus t_{\alpha}\right)
$$

on each residue disk $] z_{0}[$. Our assertion follows from this formula since

$$
\begin{aligned}
\left.\Xi(\alpha z)\right|_{] z_{0}[} & :=-\log _{p} \widehat{\theta}_{\alpha z_{0}}([\alpha] t)-\frac{1}{12} \log _{p} \Delta \\
& =-\sum_{t_{\alpha} \in E[\alpha]} \log _{p} \widehat{\theta}_{z_{0}}\left(t \oplus t_{\alpha}\right)-\frac{N \alpha}{12} \log _{p} \Delta=\left.\sum_{z_{\alpha} \in E[\alpha]} \Xi\left(z+z_{\alpha}\right)\right|_{] z_{0}[}
\end{aligned}
$$

on each residue disk.

We now prove the $p$-adic second limit formula. 
Proof of Theorem 1.2. By the definition of $\Xi(z)$ and (19), the derivative of $\Xi(z)$ is equal to $-\widehat{F}_{z_{0}, 1}(t)$ on the residue disk $] z_{0}[$. By Definition 4.10, the derivative of

$$
K_{0}^{\mathrm{col}}(0, z, 1):=E_{1,1}^{\mathrm{col}}(z)
$$

is equal to $E_{0,1}^{\mathrm{col}}(z):=-F_{1}^{\mathrm{col}}(z)$, and hence coincides with $-\widehat{F}_{z_{0}, 1}(t)$ on $] z_{0}[$. This implies that $c(z):=\Xi(z)-E_{1,1}^{\text {col }}(z)$ is a constant on the residue disk $] z_{0}[$. By Corollary 5.5 and the definition of $E_{1,1}^{\mathrm{col}}(z)$, the locally constant function $c(z)$ satisfies the distribution relation. For any torsion point $z_{0}$ of order $\mathfrak{f}$, we take $N$ such that $\pi^{N} \equiv 1 \bmod \mathfrak{f}$. Then $\left.\left[\pi^{N}\right]^{*}(] z_{0}[)=\right] z_{0}[$ and

$$
\left.\left[\pi^{N}\right]^{*} c(z)\right|_{z_{0}[}=\left.\sum_{w \in E\left[\pi^{N}\right]} c(z+w)\right|_{] z_{0}[}
$$

Since $\left.c(z)\right|_{z_{0}[}$ is constant, the above relation shows that $\left.c(z)\right|_{] z_{0}[}=0$.

The above result shows in particular that

$$
\Xi(z)=-\log _{p} \theta(z)-\frac{1}{12} \log _{p} \Delta
$$

is in fact a Coleman function.

\section{3. $p$-adic Eisenstein-Kronecker-Lerch series}

We now give the definition of the $p$-adic Eisenstein-Kronecker-Lerch series and then prove Proposition 1.3, which is a $p$-adic analogue of the first Kronecker limit formula. We will prove the proposition by considering $p$-adic counterparts of our proof in Section 2 of the classical case.

Let $p \geq 5$ be a prime of good ordinary reduction for $E$, and fix a prime $\mathfrak{p}$ of $\mathcal{O}_{K}$ over $p$. We defined in [5, Section 3.1] a $p$-adic measure $\mu:=\mu_{0,0}$ on $\mathbb{Z}_{p} \times \mathbb{Z}_{p}$ interpolating the Eisenstein-Kronecker numbers, or more precisely, the special values of the Eisenstein-Kronecker-Lerch series $K_{a+b}^{*}(0,0, b ; \Gamma) /$ $A(\Gamma)^{a}$ for $a, b \geq 0$, where $\Gamma$ is the period lattice of $E$. We define the $p$-adic Eisenstein-Kronecker-Lerch function as in the Introduction as follows.

Definition 5.6. For any integer $a \in \mathbb{Z}$, we define the $p$-adic EisensteinKronecker-Lerch function by

$$
K_{a}^{(p)}(0,0, s):=\int_{\mathbb{Z}_{p}^{\times} \times \mathbb{Z}_{p}^{\times}}\langle x\rangle^{s-1}\langle y\rangle^{a-s} \omega(y)^{a-1} d \mu(x, y) .
$$


The $p$-adic Eisenstein-Kronecker-Lerch function is analytic in $s \in \mathbb{Z}_{p}$. The reason we view this function as a $p$-adic analogue of EisensteinKronecker-Lerch series is the following interpolation property.

Proposition 5.7. For any integer $a, b$ such that $a \geq b>0$ and $b \equiv 1$ $(\bmod p-1)$, we have

$$
\frac{K_{a}^{(p)}(0,0, b)}{\Omega_{p}^{a-1}}=(-1)^{a-1}(b-1) !\left(1-\frac{\pi^{a}}{p^{a-b+1}}\right)\left(1-\frac{\pi^{a}}{p^{b}}\right) \frac{K_{a}^{*}(0,0, b)}{A(\Gamma)^{a-b}}
$$

where $\Omega_{p}$ is a p-adic period of the formal group of $E$.

Proof. This follows from the interpolation property of the measure $\mu:=$ $\mu_{0,0}$ given in [5, Proposition 3.5].

We now give the proof of Proposition 1.3.

Proof of Proposition 1.3. We consider the function

$$
f(t):=\Omega_{p} \int_{\mathbb{Z}_{p}^{\times} \times \mathbb{Z}_{p}^{\times}} y^{-1} \exp \left(y \Omega_{p}^{-1} \lambda(t)\right) d \mu(x, y)
$$

on the $p$-adic residue disk $] 0[$ around zero. If we take the derivative of $f(t)$, the interpolation property of $\mu$ given in [5, Proposition 3.5] gives the equality

$$
\begin{aligned}
\lambda^{\prime}(t)^{-1} \frac{d}{d t} f(t) & =\int_{\mathbb{Z}_{p}^{\times} \times \mathbb{Z}_{p}^{\times}} \exp \left(y \Omega_{p}^{-1} \lambda(t)\right) d \mu(x, y) \\
& =\widehat{F}_{1}(t ; \Gamma)-\bar{\pi}^{-1} \widehat{F}_{1}([\pi] t ; \Gamma)-\widehat{F}_{1}(t ; \overline{\mathfrak{p}} \Gamma)+\bar{\pi}^{-1} \widehat{F}_{1}([\pi] t ; \overline{\mathfrak{p}} \Gamma)
\end{aligned}
$$

Let $E_{1,1}^{(p)}(z ; \Gamma):=E_{1,1}^{\mathrm{col}}(z ; \Gamma)-p^{-1} E_{1,1}^{\mathrm{col}}(\pi z ; \Gamma)$. Then the differential of $E_{1,1}^{(p)}(z ; \Gamma)-E_{1,1}^{(p)}(z ; \overline{\mathfrak{p}} \Gamma)$ is given by

$$
F_{1}^{\mathrm{col}}(z ; \Gamma) \omega-\frac{\pi}{p} F_{1}^{\mathrm{col}}(\pi z ; \Gamma) \omega+F_{1}^{\mathrm{col}}(z ; \overline{\mathfrak{p}} \Gamma) \omega-\frac{\pi}{p} F_{1}^{\mathrm{col}}(\pi z ; \overline{\mathfrak{p}} \Gamma) \omega
$$

which is equal to $d f(t)$ on $] 0\left[\right.$, hence the function $f(t)-E_{1,1}^{(p)}(z ; \Gamma)+$ $E_{1,1}^{(p)}(z ; \overline{\mathfrak{p}} \Gamma)$ is a constant on the residue disk $] 0\left[\right.$. By the definition of $E_{1,1}^{(p)}$ and substituting $z=\lambda(t)$ into (16), we have the distribution relation

$$
\sum_{t_{\pi} \in E[\pi]} \widehat{E}_{1,1}^{(p)}\left(t \oplus t_{\pi}\right)=0
$$


Furthermore, since the power series $\exp \left(\Omega_{p}^{-1} \lambda(t)\right)$ gives a homomorphism of formal groups $\widehat{E}$ and $\widehat{\mathbb{G}}_{m}$ isomorphically mapping $E[\pi]$ to the group of $p$ th root of unity (see [7, Section 2.2]), we have for each $y \in \mathbb{Z}_{p}^{\times}$the equality

$$
\left.\sum_{t_{\pi} \in E[\pi]} \exp \left(y \Omega_{p}^{-1} \lambda(t)\right)\right|_{t=t \oplus t_{\pi}}=\exp \left(y \Omega_{p}^{-1} \lambda(t)\right) \sum_{t_{\pi} \in E[\pi]} \exp \left(y \Omega_{p}^{-1} \lambda\left(t_{\pi}\right)\right)=0
$$

This gives by definition (24) of $f(t)$ the distribution relation $\sum_{t_{\pi} \in E[\pi]} f(t \oplus$ $\left.t_{\pi}\right)=0$. Since both $E_{1,1}^{(p)}$ and $f(t)$ satisfy the same distribution relation, the same argument as that in the proof of Theorem 1.2 shows that we must have $f(t)=E_{1,1}^{(p)}(z ; \Gamma)-E_{1,1}^{(p)}(z ; \overline{\mathfrak{p}} \Gamma)$ on $] 0[$. On the other hand, the $p$-adic second limit formula shows that

$$
E_{1,1}^{(p)}(z ; \Gamma)=\log _{p} \theta(z ; \Gamma)-\frac{1}{p} \log _{p} \theta(\pi z ; \Gamma)+\frac{1}{12}\left(1-\frac{1}{p}\right) \log _{p} \Delta(\Gamma) .
$$

Noting that

$$
\log _{p} \theta(z ; \Gamma)-\log _{p} \theta(z ; \overline{\mathfrak{p}} \Gamma)=\log _{p}(\theta(z ; \Gamma) / \theta(z ; \overline{\mathfrak{p}} \Gamma))
$$

for $z=0$ is equal to $\log _{p}(1)=0$ and $\Delta(\overline{\mathfrak{p}} \Gamma)=\bar{\pi}^{-12} \Delta(\Gamma)$, we have

$$
f(0)=E_{1,1}^{(p)}(z ; \Gamma)-\left.E_{1,1}^{(p)}(z ; \overline{\mathfrak{p}} \Gamma)\right|_{z=0}=\left(1-\frac{1}{p}\right) \log _{p} \bar{\pi}
$$

Our assertion now follows from the fact that $f(0)=\Omega_{p} K_{0}^{(p)}(0,0,1)$.

REMARK 5.8. In the interpolation formula of (23), if we let $a=0$ and $b=1$, then the interpolation factor of the right-hand side vanishes. Hence the value

$$
\Omega_{p} K_{0}^{(p)}(0,0,1)=\int_{\mathbb{Z}_{p}^{\times} \times \mathbb{Z}_{p}^{\times}} y^{-1} d \mu(x, y)
$$

is in some sense not the constant term 0 but the residue at $s=1$ of the $p$-adic analogue of $\sum_{\gamma \in \Gamma}^{*} 1 /|\gamma|^{2 s}$. Because of this fact, the formula of Proposition 1.3 is not a perfect $p$-adic analogue of the classical first Kronecker limit formula.

Acknowledgment. This research was supported in part by Japan Society for the Promotion of Science (JSPS) grants 21674001 and 26247004. 


\section{REFERENCES}

[1] K. Bannai, Rigid syntomic cohomology and p-adic polylogarithms, J. Reine Angew. Math. 529 (2000), 205-237. MR 1799937. DOI 10.1515/crll.2000.097.

$[2] \longrightarrow$, On the p-adic realization of elliptic polylogarithms for CMelliptic curves, Duke Math. J. 113 (2002), 193-236. MR 1909217. DOI 10.1215/S0012-7094-02-11321-0.

[3] Specialization of the p-adic polylogarithm to $p$-th power roots of unity, Doc. Math. 2003, Extra Vol., 73-97. MR 2046595.

[4] K. Bannai and S. Kobayashi, "Algebraic theta functions and Eisenstein-Kronecker numbers" in Proceedings of the Symposium on Algebraic Number Theory and Related Topics, RIMS Kôkyûroku Bessatsu B4, Res. Inst. Math. Sci. (RIMS), Kyoto, 2007, 63-77. MR 2402003.

[5] - Algebraic theta functions and p-adic interpolation of EisensteinKronecker numbers, Duke Math. J. 153 (2010), 229-295. MR 2667134. DOI 10.1215/00127094-2010-024.

[6] K. Bannai, S. Kobayashi, and T. Tsuji, "Realizations of the elliptic polylogarithm for CM elliptic curves" in Algebraic Number Theory and Related Topics (2007), RIMS Kôkyûroku Bessatsu B12, Res. Inst. Math. Sci. (RIMS), Kyoto, 2009, 3350. MR 2605771.

[7] - On the de Rham and p-adic realizations of the elliptic polylogarithm for CM elliptic curves, Ann. Sci. Éc. Norm. Supér. (4) 43 (2010), 185-234. MR 2662664.

[8] A. Beilinson and A. Levin, "The elliptic polylogarithm" in Motives (Seattle, 1991), Proc. Sympos. Pure Math. 55, Amer. Math. Soc., Providence, 1994, 123-190. MR 1265553.

[9] P. Berthelot, Finitude et pureté cohomologique en cohomologie rigide, with an appendix by A. J. de Jong, Invent. Math. 128 (1997), 329-377. MR 1440308. DOI $10.1007 / \mathrm{s} 002220050143$.

[10] A. Besser, "Syntomic regulators and p-adic integration, I: Rigid syntomic regulators" in Conference on p-adic Aspects of the Theory of Automorphic Representations (Jerusalem, 1998), Israel J. Math. 120, 2000, 291-334. MR 1809626. DOI 10.1007/BF02834843.

[11] - "Syntomic regulators and $p$-adic integration, II: $K_{2}$ of curves" in Conference on p-adic Aspects of the Theory of Automorphic Representations (Jerusalem, 1998), Israel J. Math. 120, 2000, 335-359. MR 1809627. DOI 10.1007/BF02834844.

[12] - Coleman integration using the Tannakian formalism, Math. Ann. 322 (2002), 19-48. MR 1883387. DOI 10.1007/s002080100263.

[13] A. Besser and R. de Jeu, The syntomic regulator for the K-theory of fields, Ann. Sci. Éc. Norm. Supér. (4) 36 (2003), 867-924. MR 2032529. DOI 10.1016/j.ansens.2003.01.003.

[14] R. F. Coleman, Dilogarithms, regulators and p-adic L-functions, Invent. Math. 69 (1982), 171-208. MR 0674400. DOI 10.1007/BF01399500.

[15] R. Damerell, L-functions of elliptic curves with complex multiplication, I. Acta Arith. 17 (1970) 287-301. MR 0285540.

[16] - L-functions of elliptic curves with complex multiplication, II. Acta Arith. 19 (1971), 311-317. MR 0399103.

[17] P. Deligne, "Le groupe fondamental de la droite projective moins trois points" in Galois Groups Over $\mathbb{Q}$ (Berkeley, 1987), Math. Sci. Res. Inst. Publ. 16, Springer, New York, 1989, 79-297. MR 1012168. DOI 10.1007/978-1-4613-9649-9_3. 
[18] E. de Shalit, Iwasawa Theory of Elliptic Curves with Complex Multiplication: p-adic L Functions, Perspect. Math. 3, Academic Press, Boston, 1987. MR 0917944.

[19] H. Furusho, p-adic multiple zeta values, I: p-adic multiple polylogarithms and the p-adic KZ equation, Invent. Math. 55 (2004), 253-286. MR 2031428. DOI 10.1007/s00222-003-0320-9.

[20] N. M. Katz, p-adic interpolation of real analytic Eisenstein series, Ann. of Math. (2) 104 (1976), 459-571. MR 0506271.

[21] A. Levin, Elliptic polylogarithms: An analytic theory, Compos. Math. 106 (1997), 267-282. MR 1457106. DOI 10.1023/A:1000193320513.

[22] G. Robert, Unités elliptiques et formules pour le nombre de classes des extensions abéliennes d'un corps quadratique imaginaire, Mém. Soc. Math. France 36, Soc. Math. France, Paris, 1973. MR 0469889.

[23] M. Somekawa, Log-syntomic regulators and p-adic polylogarithms, K-Theory $\mathbf{1 7}$ (1999), 265-294. MR 1703301. DOI 10.1023/A:1007755726476.

[24] A. Weil, Elliptic Functions According to Eisenstein and Kronecker, Ergeb. Math. Grenzgeb (3) 88, Springer, Berlin, 1976. MR 0562289.

[25] J. Wildeshaus, On an elliptic analogue of Zagier's conjecture, Duke Math. J. 87 (1997), 355-407. MR 1443532. DOI 10.1215/S0012-7094-97-08714-7.

[26] D. Zagier, Periods of modular forms and Jacobi theta functions, Invent. Math. 104 (1991), 449-465. MR 1106744. DOI 10.1007/BF01245085.

Kenichi Bannai

Department of Mathematics

Keio University

Yokohama 223-8522

Japan

bannai@math.keio.ac.jp

Hidekazu Furusho

Graduate School of Mathematics

Nagoya University

Nagoya 464-8602

Japan

furusho@math.nagoya-u.ac. jp

Shinichi Kobayashi

Mathematical Institute

Tohoku University

Sendai 980-8578

Japan

shinichi@math.tohoku.ac.jp 